\title{
Decorrelating Action of Inhibition in Neocortical Networks
}

\author{
Tanya Sippy and Rafael Yuste \\ HHMI, Department of Biological Sciences, Columbia University, New York, New York 10027
}

Inhibitory GABAergic interneurons have been extensively studied but their contribution to circuit dynamics remain poorly understood. Although it has been suggested that interneurons, especially those belonging to the same subclass, synchronize their activity and impart this synchrony onto their local network, recent theoretical and experimental work have challenged this view. To better understand the activity of interneurons during cortical activity, we combined molecular identification, two-photon imaging, and electrophysiological recordings in thalamocortical slices from mouse somatosensory cortex. Using calcium imaging to monitor cortical activity, we found low spiking correlations among parvalbumin or somatostatin interneurons during cortical UP states, indicating that interneurons do not synchronize their firing. Intracellular recordings confirmed that nearby interneurons do not display more synchronous spiking than excitatory cells. The lack of interneuron synchrony was also evident during slow oscillations, even among interneurons that were electrically coupled via gap junctions, suggesting that their coupling does not function to synchronize their activity. Using voltage-clamp recordings from nearby pyramidal cells, we found that inhibitory currents (IPSCs) are more correlated than excitatory ones, but that correlated IPSCs arise from the activation of common presynaptic inhibitory cells, rather than from synchronization of interneuron activity. Finally, we demonstrate that pharmacologically reducing inhibitory currents increases correlated excitatory activity. We conclude that inhibitory interneurons do not have synchronous activity during UP states, and that their function may be to decorrelate rather than to synchronize the firing of neurons within the local network.

\section{Introduction}

Information coding in neural networks probably depends on the rate of action potential firing (rate code) and the precise timing of spikes (temporal code) across population of neurons. This code is not just the property of a single neuron, which by itself has limited capacity to carry information. Instead, the relevant computations to explain perception or behavior must be a property of the joint functioning of many neurons (McClurkin et al., 1991). In fact, synchrony, the most basic temporal relationship among two or more neurons, has been widely observed in the CNS. Different modes of synchrony have been described both in vitro and in vivo, and are thought to play a role in development, sensory perception, motor control and other forms of cognition, such as attention (Sanes, 2003; Uhlhaas et al., 2010; Bruno, 2011; Gordon, 2011).

Based on many studies in both cortex and hippocampus it is widely accepted that inhibitory transmission is necessary for many forms of synchronous activity (Bartos et al., 2002). In fact,

\footnotetext{
Received Sept. 26, 2012; revised April 21, 2013; accepted April 24, 2013.

Author contributions: T.S. and R.Y. designed research; T.S. performed research; T.S. analyzed data; T.S. and R.Y. wrote the paper.

This work was supported by the Kavli Institute for Brain Science, NEI, NINDS, NIHM, NIDA, Keck Foundation and NARSAD. This material is based on work supported by, or in part by, the US Army Research Laboratory and the US Army Research Office under contract number W911NF-12-1-0594. We thank A. Packer, A. Woodruff, and T. Machado for helpful feedback, Y. Shin for anatomical reconstructions, M. Karnani, A. Pala, and C. Petersen for reagents and technical assistance with immunohistochemistry, and members of the Yuste laboratory for help and comments.

The authors declare no competing financial interests.

Correspondence should be addressed to Tanya Sippy, École Polytechnique Fédérale de Lausanne, SV-BMI-LSENS Station 19, CH-1015 Lausanne, Switzerland. E-mail: missippy@gmail.com.

DOI:10.1523/JNEUROSCI.4579-12.2013

Copyright $\odot 2013$ the authors $\quad 0270-6474 / 13 / 339813-18 \$ 15.00 / 0$
}

it is known that interneurons that belong to the same subtype are commonly electrically coupled (Galarreta and Hestrin, 1999; Gibson et al., 1999), and this is thought to lead to synchronous activity (Gentet et al., 2010), and to promote neuronal oscillations (Deans et al., 2001; Gibson et al., 2005; Kaminski et al., 2011). Nevertheless, most studies focusing on coupling of interneurons examined at most two neurons in very close proximity (within $200 \mu \mathrm{m}$ ), and it is unclear how such coupling affects spiking of larger populations within and beyond these distances. Moreover, interneurons that normally display correlated firing in cerebellum, can become rapidly and strongly desynchronized in response to synaptic inputs and impart this desynchronization onto the local network (Vervaeke et al., 2010).

We investigated the function of subpopulations of interneurons, and address what effect inhibition has on activity of excitatory cells within local circuits. For this task, we chose to use a thalamocortical slice preparation in which recurrent cortical activity (UP states) can be thalamically triggered, or occur spontaneously, either in individual events, or in an oscillatory fashion. We find, first, that interneurons, even those that belong to the same cell class (parvalbumin or somatostatin) do not have more correlated activity than their excitatory counterparts. Second, even among electrically coupled interneurons, spiking is not synchronous during spontaneous and evoked cortical activity. Third, although isolated IPSCs are more correlated than EPSCs, this can be explained by the firing of individual interneurons that are presynaptic to neighboring pyramidal cells, rather than by synchronized firing. Finally, a mild pharmacological reduction of inhibition can significantly increase excitatory correlations. Together, these results provide evidence that interneurons belonging to the same cell class do not coordinate their firing, and open 
the possibility of other functions of inhibitory neurons other than synchronization of the network.

\section{Materials and Methods}

Slice preparation. Thalamocortical slices, $400 \mu \mathrm{m}$ thick, were prepared from postnatal day 13 (P13) to $\mathrm{P} 18$, or p28-35 (as indicated in text) from GIN (Oliva et al., 2000) or G42 (Chattopadhyaya et al., 2004) transgenic mice of either sex, as previously described (MacLean et al., 2005). Slices were cut with a vibratome (VT1200S; Leicaor Microm 650 V, ThermoFisher Scientific) in ice-cold oxygenated modified ACSF that included $0.5 \mathrm{~mm} \mathrm{CaCl}_{2}$ and $3.5 \mathrm{~mm} \mathrm{MgSO}_{4}$, in which $\mathrm{NaCl}$ was replaced by an equimolar concentration of sucrose. Experiments were performed with ACSF containing the following (in mM): $123 \mathrm{NaCl}, 3 \mathrm{KCl}, 26 \mathrm{NaHCO}_{3}, 1$ $\mathrm{NaH}_{2} \mathrm{PO}_{4}, 2 \mathrm{CaCl}_{2}, 2 \mathrm{MgSO}_{4}$, and 10 dextrose, which was continuously aerated with $95 \% \mathrm{O}_{2}, 5 \% \mathrm{CO}_{2}$, and perfused over the slice at high perfusion rates $(125-175 \mathrm{ml} / \mathrm{h})$. In a subset of experiments, the ACSF was modified to include $1.2 \mathrm{mM} \mathrm{CaCl}_{2}$ and $1.0 \mathrm{mM} \mathrm{MgSO}_{4}$ and either $3.5 \mathrm{~mm}$ $\mathrm{KCl}$ or $5.0 \mathrm{~mm} \mathrm{KCl}$. For experiments using gabazine (GZ), 100-200 nm concentrations were used.

Electrophysiology. Thalamocortical projection neurons were activated using bipolar platinum-iridium electrodes (No. CE2C55, Frederick Haer) placed in the ventrobasal nucleus (VB) of the thalamus. Stimuli were $200 \mu \mathrm{s}$ in duration, $20-100 \mu \mathrm{A}$ in amplitude and were applied individually or as a train of $4-8$ stimuli, each separated by $25 \mathrm{~ms}(40 \mathrm{~Hz})$ using a Master 8 pulse generator coupled to a Iso-flex stimulator (AMPI). For each slice the minimal pulse amplitude necessary to evoke recurrent activity was used which allowed us to minimize potential activation of corticothalamic neurons (Ferster and Lindstrom, 1985; Agmon and Connors, 1991). All recordings were made at $33^{\circ} \mathrm{C}$. Calcium imaging of populations of neurons (Yuste and Katz, 1991) was used to identify online responding cells in layer $2 / 3$, and these neurons were then targeted for whole-cell recording. Whole-cell current-clamp recordings using Multiclamp 700B amplifiers (Molecular Devices) were made from neurons in layer 4 using $4-6 \mathrm{M} \Omega$ micropipettes, filled with the following (in $\mathrm{mm}$ ): $130 \mathrm{~K}$ methylsulfate, $2 \mathrm{MgCl}_{2}, 0.6 \mathrm{EGTA}, 10 \mathrm{HEPES}, 4 \mathrm{ATP}-\mathrm{Mg}$, and 0.3 GTP-Tris, pH 7.2 (290-295 mOsm). To characterize neurons, $500-1000 \mathrm{~ms}$ depolarizing DC current injections were given to each cell and resultant action potential firing patterns were analyzed, following the Petilla convention nomenclature (Ascoli et al., 2008).

Morphological processing. Neurons were filled with biocytin by diffusion from the intrapipette solution during recordings, with electrodes containing $0.4 \mathrm{~g} / 100 \mathrm{ml}$ of biocytin in addition to the solution described above. At the end of each recording, slices were fixed overnight in $4 \%$ paraformaldehyde. Thereafter, slices were rinsed several times in $0.12 \mathrm{M}$ PBS. Slices were then transferred to $30 \%$ sucrose in $15 \mathrm{ml}$ of $0.12 \mathrm{M}$ PBS for at least $2 \mathrm{~h}$ and as long as 1 week. Slices were then frozen in an embedding medium. After freezing, slices were rinsed in $0.12 \mathrm{M}$ PBS several times. Slices were then incubated in $1 \% \mathrm{H}_{2} \mathrm{O}_{2}$ in $0.12 \mathrm{M}$ PBS for 30 min under agitation and rinsed in $0.12 \mathrm{M}$ PBS once for $15 \mathrm{~min}$. After two other washes in $0.02 \mathrm{M} \mathrm{KPBS}$, the slices were incubated overnight under agitation in 1\% avidin-biotin complex (ABC Kit Standard, Vector Laboratories) prepared in $0.3 \%$ Triton X-100. After three rinses in phosphate buffer, biocytin was revealed by diaminobenzidine. After two final rinses in phosphate buffer, slices were mounted onto slides. The neurons were reconstructed with Neurolucida (Micro Bright Field).

Antibody labeling. Anesthetized mice (p14) were perfused transcardially with $4 \%$ paraformaldehyde in $0.1 \mathrm{~m}$ phosphate buffer, $\mathrm{pH} 7.4$. A vibratome (Leica VT1000 s) was used to cut $50 \mu \mathrm{m}$ thick coronal sections from somatosensory cortex. Sections were blocked in $10 \%$ normal donkey serum for $1 \mathrm{~h}$ at RT, then incubated $48 \mathrm{~h}$ at RT with primary antibodies: polyclonal rabbit anti-PV (1:1000, Swant PV25) and goat anti-GFP (1:3000; Abcam 5450) in 5\% normal donkey serum and $0.3 \%$ Triton. Subsequently, sections were incubated with anti-rabbit CY3 IgG (1: 500; Jackson ImmunoResearch) and anti-goat Alexa488 (Invitrogen) and mounted.

Images from layer $2 / 3-5$ of $S 1$ were acquired using $20 \times$ air objective with a confocal microscope (Zeiss 700 LSM). No bleed through between 488 and 555 channels was observed. Scans were collected in sequential mode and later merged. Images were saved as TIF files and analyzed with MacBioPhotonics ImageJ.

Calcium indicator bulk loading and imaging. Slices were bulk loaded with Fura 2-AM for visualization of action potential-related activity in neuronal somata. Slices were placed onto the bottom of a small Petri dish $(35 \times 10 \mathrm{~mm})$ filled with a vortexed mixture of $2 \mathrm{ml}$ of ACSF, an aliquot of $50 \mu \mathrm{g}$ Fura 2-AM (Invitrogen), $15 \mu \mathrm{l}$ DMSO, and $2 \mu \mathrm{l}$ Pluronic F-127 (Invitrogen). A cover was placed over the Petri dish and it was incubated in the dark at $35-37^{\circ} \mathrm{C}$ and oxygenated by puffed $\mathrm{CO}_{2} / \mathrm{O}_{2}$ gas for $\sim 25$ $\mathrm{min}$. To locate regions in the cortex connected to the area of thalamus we stimulated, we first imaged at low $(4 \times)$ magnification. Barrels were identified in bright field as repeating "hollow rectangles", corresponding to regions of high cell density, occurring in layer 4 , as confirmed with cytochrome oxidase staining (Feldmeyer et al., 1999). The region in the barrel fields which responded earliest to stimulation was then chosen for higher cell resolution imaging and patch-clamping.

Changes in intracellular free $\mathrm{Ca}^{2+}$ were visualized with a high numerical aperture $20 \times(0.95)$ Olympus Plan FL objective with an upright fluorescence microscope (Olympus BX50WI; Olympus Optical) using a Ti:sapphire laser (Chameleon Ultra II, Coherent; $>3 \mathrm{~W}, 140$ fs pulses, 80 $\mathrm{MHz}$ repetition rate) tuned to either 790 (fura-2 AM imaging) or $900 \mathrm{~nm}$ (GFP imaging). A Hamamatsu C9100-12 camera and Micro-Manager (Vale Labs) and ImageJ software (a public domain, Java-based image processing program developed at the National Institutes of Health) were used for targeting neurons for imaging activity from populations of neurons. Frames were acquired at 15-15.67 ms/frame. Binning was performed such that images were $256 \times 256$ pixels. Files were saved as multipage TIFF stacks.

First, a slow raster scan was performed at a low frame rate $(1 \mathrm{~Hz})$ to identify cell bodies. In G42 and GIN transgenic knock-in mice, the GABAergic GFP labeled interneurons were excited at $900 \mathrm{~nm}$. Subsequently, the same field was imaged at $790 \mathrm{~nm}$ to visualize loaded cell bodies. After these imaged were acquired with the camera, neurons were targeted for imaging on their cell bodies, using a spatial light modulator (SLM). We use a model 1080P phase SLM from Holoeye, which has a resolution of $1920 \times 1080$ pixels, and an 8-bit phase quantization, with a $60 \mathrm{~Hz}$ refresh rate. Patterns were generated with software from Holoeye. In our microscope, collimated light from our laser passes through an optional Pockels cell, which regulates total power, and after beam reshaping and resizing, hits the reflective SLM. A system of lenses relays the image of the SLM surface to the back aperture of the main microscope objective. Some small fraction $(<25 \%)$ of the incoming light remains undiffracted; this is the "zero-order" beam. We used an "on-center" configuration wherein the nondiffracted beam is present in the FOV, and we employ a small beam-stop to remove it.

Image analysis. To detect calcium signals from imaged cells, loaded neurons were automatically identified using a custom written ImageJ plug-in (written by T.A. Machado) on the raw image of the slice, and then the fluorescence of these cells was measured as a function of time. All remaining image processing was performed using custom written software in MATLAB (MathWorks).

Fluorescence traces were then preprocessed. Because some slow drift was sometimes present in the traces, each trace was Fourier transformed, and all frequencies $<0.5 \mathrm{~Hz}$ were set to zero $(0.5 \mathrm{~Hz}$ was chosen by eye); the resulting fluorescence trace was then normalized to be between zero and one. Taking advantage of the high temporal resolution of our data, we used a fast nonnegative deconvolution algorithm (Vogelstein et al., 2010) to infer the approximately most likely spike train underlying our fluorescence data. Briefly, the algorithm uses a model that assumes somatic fluorescence arising from the calcium indicator can be approximated by convolving the neuron's spike train with an exponentially decaying kernel. Noise is assumed to be Gaussian, and the spike train is assumed to be Poisson. Given this model, and assuming the Poisson spike train can be well approximated as an exponential, a convex objective function can be derived. The objective function was numerically optimized given a nonnegativity constraint on the spike train implemented using a barrier term. Parameters were manually determined and not estimated from the data. Spike trains were deconvolved from all putative neurons with at least a $5 \% \Delta \mathrm{F} / \mathrm{F}$ change within one movie we 
stored them in a matrix Finally, we correlated the vectors, each representing the estimated spike train from a single contour, using the MATLAB built in function CORRCOEF.

Although fura 2-AM preferentially labels neurons, any contour with long rise times ( $>2$ frames), and slow decay times, typical of astrocyte calcium signaling were excluded from the analysis.

Electrophysiology analysis. UP states were detected automatically (based on an algorithm written by B.O. Watson) from whole-cell current-clamp traces based on fulfillment of the following minimum criteria: at least $500 \mathrm{~ms}$ of depolarization of $3 \mathrm{mV}$ or more and at least 3 action potentials during this depolarization. If the neuron did not fire action potentials, a continuous depolarization of $5 \mathrm{mV}$ for a minimum of $500 \mathrm{~ms}$ was required. This allowed us to detect all UP states despite the variability of membrane behavior exhibited by different neurons. Simultaneous patch-clamp recordings confirmed that these criteria allowed for the reliable detection of network UP state events which occurred simultaneously in simultaneously recorded cells. Further, after automatic detection, all events meeting these requirements were reviewed by the experimenter and could be rejected at that point. Durations and amplitudes for verified UP states were quantified based on automatically detected UP state start times and stop times. Action potentials were detected based on their amplitudes and durations and numbers within detected UP states were quantified.

Cross-correlograms and were computed using MATLAB 7.11.0 (MathWorks). Shufflegrams were computed by correlating each UP state segment with a randomly selected UP state segment in the same cell without replacement. For calculation of correlations, 300-500 ms periods of activity were randomly selected by the computer program and averaged together to get the estimated correlation for each cell. For reshuffling of spike times, only spikes occurring within the first $800 \mathrm{~ms}$ of the UP state were randomly redistributed, and the minimum intercell spike interval was recalculated.

Using paired recordings, monosynaptic connections were detected by evoking single action potentials (at $0.1 \mathrm{~Hz}$ ) in postsynaptic neurons by examining the latency, amplitude, rise time, and failure rate of recorded evoked IPSCs (for inhibitory connections). These values were recorded at $0 \mathrm{mV}$ with a chloride reversal potential of $-70 \mathrm{mV}$. For PC to PC pairs, single action potentials were similarly evoked, with the resulting EPSC recorded in the postsynaptic cell at $-70 \mathrm{mV}$. Twenty-five trials were subsequently averaged for each connected pair.

Analysis of IPSCs and EPSCs was performed using event detector programs (Synaptosoft). The amplitude of the events had to exceed a detection threshold set at the maximum limit of the noise (usually $<8 \mathrm{pA}$ ). Rise times were measured from 10 to $90 \%$ of peak amplitude.

Statistics were implemented using InStat (GraphPad). Briefly, a Student's $t$ test was implemented for all comparisons, unless the distributions failed a normality test, in which case a Mann-Whitney was used. In cases where there was a comparison of two or more datasets, a one-way ANOVA was used, and whether the data could not be assumed to be sampled from Gaussian distributions, a Kruskal-Wallis was used. For bin-by-bin comparisons of $>2$ samples, a repeated-measures ANOVA was used unless the data did not follow a Gaussian distribution in which case a Friedman test was used. Unless otherwise noted, all measurements are expressed as mean \pm SEM.

\section{Results}

Experiments were conducted in G42 and GIN GAD67-GFP transgenic mouse lines, in which parvalbumin-expressing inhibitory (PV)-positive and somatostatin-expressing inhibitory (SOM)-positive neurons, respectively, are labeled with GFP throughout the cortex (Oliva et al., 2000; Chattopadhyaya et al., 2004). Although together these two populations are thought to represent the majority of the neocortical interneurons (Xu et al., 2010; Rudy et al., 2011), it is important to note that in each of these mouse lines only a subset of these population of neurons are labeled with GFP. We have previously shown that GFP-positive cells in layer $2 / 3$ of the GIN mouse line are a relatively homogeneous population of Martinotti cells (Fino and Yuste, 2011), but
PV cells are more diverse, including basket cells, and chandelier cells, among others (Kozloski et al., 2001; Markram et al., 2004; Woodruff et al., 2009). Furthermore, in visual cortex (V1) of young G42 mice some GFP cells are PV-negative (Buchanan et al., 2012).

To characterize the identity of GFP-labeled neurons in somatosensory cortex (S1) of G42 mice we immunostained GFPpositive cells for PV. In layer 2/3, we found good concordance between GFP and PV staining (Fig. 1A) (91.2 $\pm 3.4 \%$ of cells that were GFP-positive were PV-positive; $n=3$ animals), similar to results reported in mature animals (Chattopadhyaya et al., 2004). Interestingly, in layers 4 and 5 this concordance dropped significantly (Fig. 1, $A 2, A 3, B$ ) (layer 4: $71.5 \pm 5.8 \%$, layer 5: $73.4 \pm 6.5 \%$; $n=3$ animals, $p<0.01$ one-way ANOVA, $\mathrm{L} 4$ vs L $5 p>0.05, \mathrm{~L} 2 / 3$ vs L4 and L2/3 vs L5 $p<0.01$ Tukey-Kramer multiple-comparisons test). Finally, the percentage of GFP-positive cells of all labeled PV cells (or sparseness of the labeling) differed by layer, and was significantly smaller in layer 4 than either layers $2 / 3$ or 5 (Fig. 1C) (39.6 \pm $4.1 \%$ in $\mathrm{L} 4$ vs $83.2 \pm 6.8 \%$ in $\mathrm{L} 2 / 3$ and $78.4 \pm 7.3 \% \mathrm{~L} 5, n=3$ animals, $p<0.001$ one-way ANOVA; L2/3 vs L4 and L4 vs L5 $p<$ 0.001; L2/3 vs L5 $p>0.05$ Tukey-Kramer multiple-comparisons test). Because the colocalization of GFP and PV increases (and the sparseness of labeling decreases) throughout development, it is likely that PV expression matures with age and that the GFP-positive PVnegative interneurons will eventually express PV (Chattopadhyaya et al., 2004; Buchanan et al., 2012). We refer to all GFP-positive neurons in the SOM and PV GAD67 mouse lines as "sGFP" and "pvGFP", respectively, whereas unlabeled cells are referred to as "GFPneg".

\section{Fast imaging of thalamically evoked activity in interneuron subtypes}

To characterize the synchronous activity of sGFP, pvGFP, and GFPneg neurons, we used thalamocortical somatosensory slices. These slices were bulk loaded with fura-2 AM (Fig. 2A), enabling two-photon calcium imaging of action potential activity of neuronal populations in layers $2 / 3$ and 4 . An SLM was used to split the two photon laser into multiple beamlets, and $40-50$ neuron cell bodies within a $300 \times 350 \mu \mathrm{m}$ field of view within layers $2 / 3$ and 4 of the barrel were targeted for fast imaging (Nikolenko et al., 2008) (Fig. 2B). The SLM obviated the need for raster scanning the laser, allowing us to take advantage of the spatial resolution and high signal-to-noise ratio two-photon imaging affords, while collecting fluorescence at frame rates of $60-66 \mathrm{~Hz}$ with an electron multiplying CCD camera (EMCCD).

We previously explored the effect of different frequencies of thalamic stimuli on cortical activity and found that highfrequency $(>10 \mathrm{~Hz})$ stimulation was necessary to cause detectable activation in barrel cortex, whereas single stimuli failed to activate large numbers of cortical ensembles (MacLean et al., 2005). As before (MacLean et al., 2005), brief trains of six stimuli at $40 \mathrm{~Hz}$, applied to the VB thalamus reliably activated groups of neurons (UP states) in both layers $2 / 3$ and 4 (Fig. 2C). A fast nonnegative deconvolution filter was used to infer the most likely spike train of each neuron given the fluorescence observations (Vogelstein et al., 2010). To empirically adjust the parameters for the algorithm, we performed cell-attached patch-clamp recordings from neurons identified during the stimulus driven cortical response. Because GFP-expressing interneurons, particularly PV-positive cells, may have different calcium buffering capacities, calibration was done in GFPneg, pvGFP, andsGFP cells (Fig. $2 D)$. For all three cell types, the algorithm performed well at estimating the likelihood of a spike in any given frame, even when 
A1

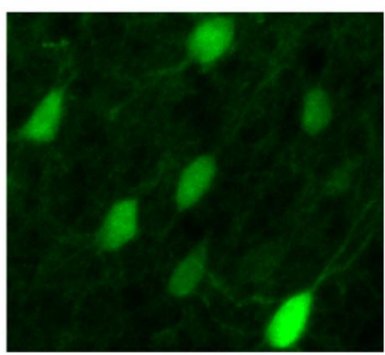

A2

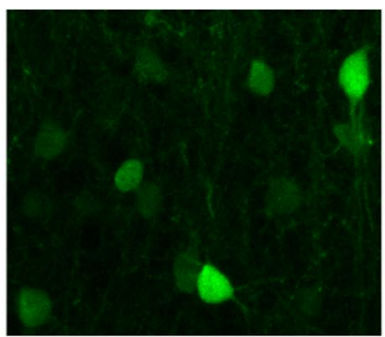

A3

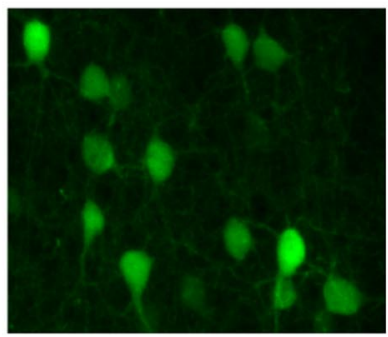

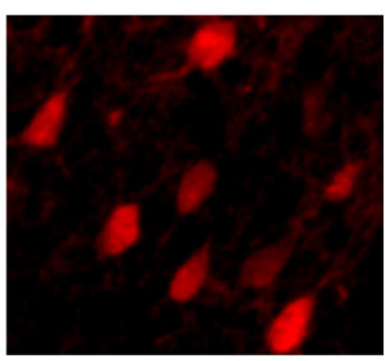
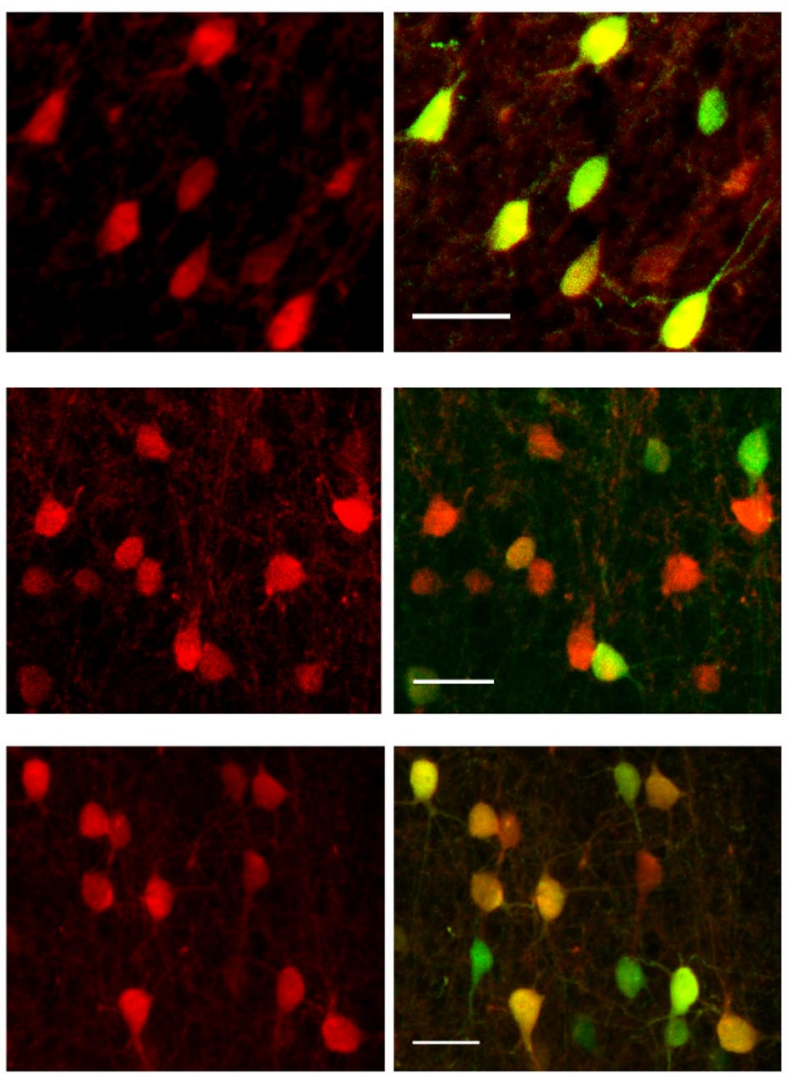

\section{B}

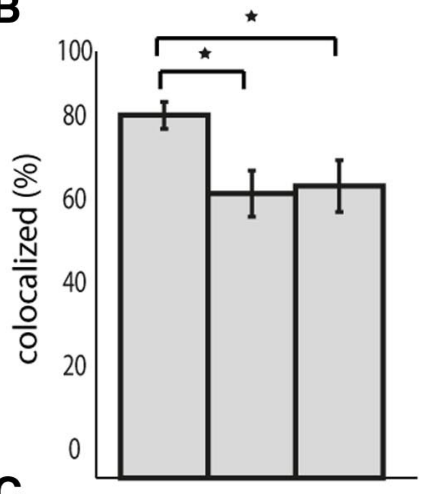

C

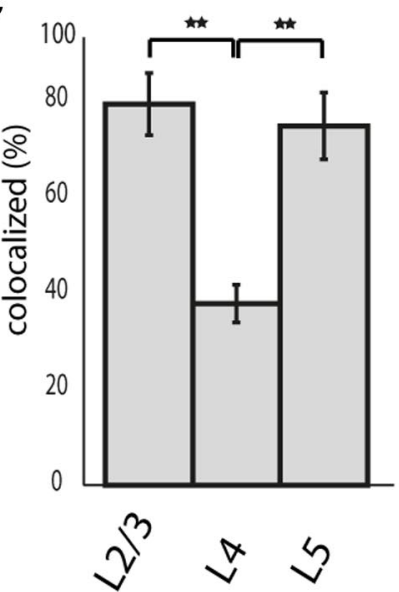

Figure 1. Layer-specific colocalization of GFP and PV in G42 animals. A1-A3, Cortical S1 was double labeled for GFP and PV. Left panel (green) shows antibody labeling for GFP, middle column shows $\mathrm{PV}$ immunolabeling (red), and far right column is the overlay. Top row is $\mathrm{L} 2 / 3$, middle row is $L 4$, and bottom row is $L 5$. Scale bar, $30 \mu \mathrm{m}$. $B$, The percentage of GFP-positive cells that were also positive for PV was significantly higher in layer $2 / 3$ than either layer 4 or $5\left(^{*} p<0.01\right.$, one-way ANOVA). $C$, The percentage of PV cells that were labeled for GFP was significantly lower in L4 than either layer $2 / 3$ or $5{ }^{* *} p<0.001$, one-way ANOVA).

we made no allowance for a window of jitter around the time of the actual spike (Fig. 2E) (GFPneg: sensitivity, $80.0 \pm 2.5 \%$, specificity, $98.1 \pm 0.5 \% n=11$; pvGFP: sensitivity $78.0 \pm 3.4 \%$ specificity $98 \% \pm 0.8 \% n=4$, sGFP: sensitivity $82 \pm 3.1 \%$ specificity $97.9 \pm 0.6 \% n=5$ ), and detected nearly all spikes within a window of \pm 1 frame (GFPneg: sensitivity $95.0 \pm 3.1 \%$, specificity, $95.5 \pm 1.4 \%$, pvGFP: sensitivity $94 \pm 4.1 \%$ specificity $96 \pm$ $0.7 \%$, sGFP: sensitivity $98.0 \pm 2.9 \%$ specificity $94.3 \pm 0.6 \%$ ). With the ability to detect single spikes with such high temporal resolution, we could address the timing of coordinated activity in subgroups of neurons at fast time scales.

\section{Activity of neocortical interneuron subtypes is not correlated}

We first performed SLM imaging of pvGFP and sGFP GABAergic interneurons to study the timing of activity in these interneurons compared with other cell types. After stimulation of the thalamus, 35-75\% of neocortical neurons were "active," or displayed at least a single $5 \%$ change in fluorescence, normalized to baseline $(\Delta \mathrm{F} / \mathrm{F})$. We typically imaged $5-15$ pvGFP or sGFP interneurons alongside 20-40 GFPneg neurons (Fig. 3A). In both the G42 and GIN transgenic mouse lines, close to $90 \%$ of GFPneg cells were excitatory as determined by their intrinsic electrophysiological properties (Peters, 1984; Gonchar et al., 2007), so we equate GFPneg with excitatory neurons. The probability of calcium activation of pvGFP cells, sGFP cells, and GFPneg was found to be similar, with no significant differences between these cell types (Fig. $3 B, C$ ) (pvGFP, $53.7 \pm 5.4 \%$; sGFP, $68.2 \pm 4.6 \%$; GFPneg, $56.4 \pm 3.3 \%$; one-way ANOVA, $p=0.08$ ).
To address whether GABAergic interneurons subpopulations display more correlated firing than other cell types, we computed correlations from the deconvolved spike time estimates, which avoids overestimating the correlation coefficient that results from computing correlations directly from the raw fluorescence traces (Smith and Häusser, 2010). We first calculated these correlation coefficients among all pairs of active pvGFP, sGFP, or GFPneg cells during thalamically stimulated UP states. The normalized distributions of the correlations between pvGFP, sGFP, and GFPneg cell pairs were similar, showing no significant differences when compared in a bin-by-bin manner (Fig. 3D) (Friedman test, $p=0.2874$ ). Whereas some cell pairs belonging to all cell types showed highly correlated activity (correlation coefficients $>0.6$ ), in general all groups showed low correlations, with no significant difference between them (correlation among pvGFP neurons, $0.14 \pm 0.01, n=196$ pairs; correlation among sGFP, $0.10 \pm 0.01, n=67$ pairs; correlation among GFPneg cells, $0.12 \pm 0.002, n=3119$ pairs, from both G42 and GIN animals; $p=0.4198$, Kruskal-Wallis test). Therefore, even when two cells belonged to the same cell class, they did not show similarities in firing when compared with other cell types.

We wondered whether there would be a significant inverse relationship between distance separating cell bodies and their activity correlations. To test this, we plotted the distance between cell bodies (in $\mu \mathrm{m}$ ) versus correlation coefficient for all cell pairs. We found, for all three groups, pvGFP, sGFP, and GFPneg no significant relationship between these two variables (Fig. $3 E$ ) (pvGFP Pearson's $r=-0.078, p=0.27$; sGFP Pearson's $r=$ 

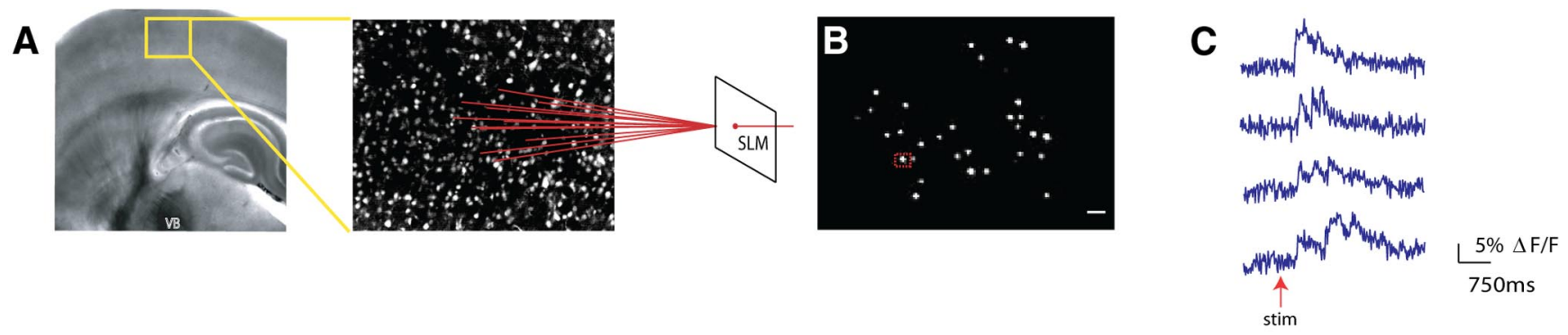

GFPneg

D

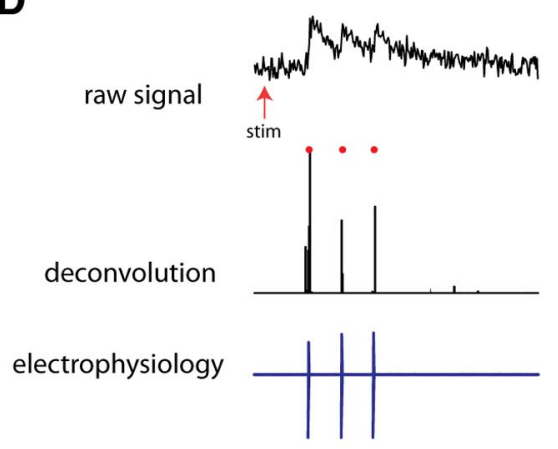

$\mathbf{E}$

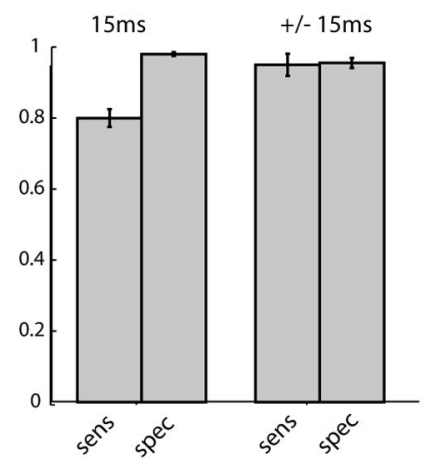

PV
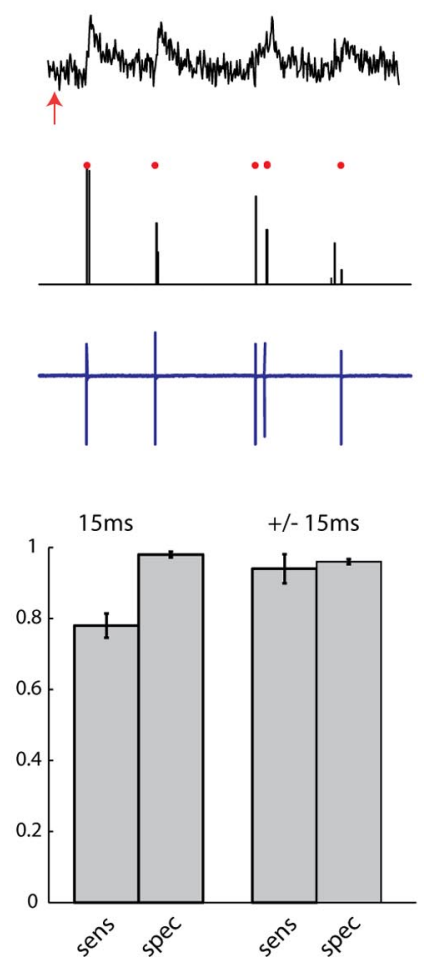

SOM
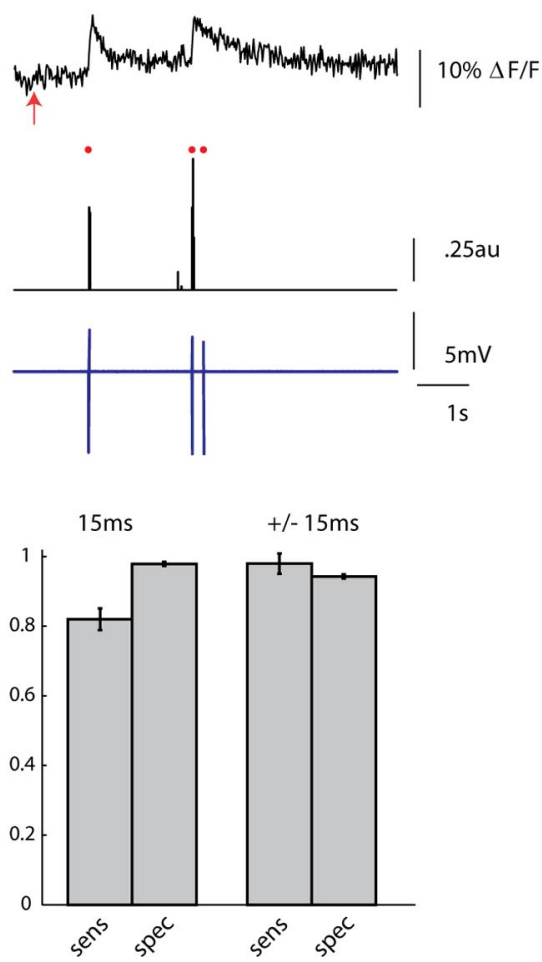

Figure 2. Two-photon fast calcium imaging with a single-spike deconvolution algorithm. $A$, Light micrograph of a $S 1$ thalamocortical slice preparation with intact thalamic input nucleus VB, thalamocortical axons, and the somatosensory cortex. A stimulating electrode is placed in VB. Superimposed yellow box indicates location, over layers $2 / 3$ and 4 , of illustrated two photon $z$ stack to right. Neurons pictured in this field are loaded with fura-2 AM dye, and targeted with an SLM (far right). $\boldsymbol{B}$, Two-photon image of a single frame showing neuronal cell bodies targeted with two photon illumination with the SLM. Cell outlined in red was targeted in cell-attached mode (D). Scale bar, $30 \mu \mathrm{m}$. C, Examples of fluorescence signals showing changes in fluorescence, normalized to baseline $(\triangle F / F)$, from four cells imaged at $66.6 \mathrm{~Hz}$ with an EMCCD. D, Three examples from calibration experiments in which a PC (left), a PV cell (middle), and SOM cell (right) were targeted for cell attached recording during simultaneous stimulation of the thalamus. Top trace shows raw fluorescence signal from that cell imaged at $66.6 \mathrm{~Hz}$. Middle trace is the deconvolution of the calcium signals using parameters obtained from electrophysiology to obtain estimated spike times. Red dots above both traces indicate the time of the actual spikes. Bottom trace shows the associated electrophysiological trace. E, Sensitivity (true-positive rate) and specificity (1-false-positive rate) of the deconvolution algorithm. These rates were calculated while allowing for either no window around each spike to search for a signal ( $15 \mathrm{~ms})$, or for a window of \pm 1 frame around each spike ( $\pm 15 \mathrm{~ms})$.

$0.0078, p=0.78$; GFPneg Pearson's $r=0.019, p=0.27)$. Therefore, neighboring neurons, even those of the same molecular subtype, do not have more correlated activity than those at further distances.

\section{Spiking synchrony of interneurons is similar to that of principal cells}

Although using calcium imaging we found that PV or SOM interneurons did not show correlated activity, we could not exclude the possibility that these neurons were significantly correlated on a time scale faster than the temporal resolution of our SLM imaging (15 ms). This seemed likely because interneurons belonging to both these subtypes have been shown to be coupled either chemically, and/or electrically via gap junctions, both of which can promote fast synchrony under certain conditions (Beierlein et al., 2000; Galarreta and Hestrin, 2001; Hu et al., 2011).

To examine this, we performed whole-cell electrophysiological recordings from 43 pvGFP interneurons in layer $2 / 3$, with biocytin in our internal solution, and performed anatomical and electrophysiological analysis of these cells. Anatomically, pvGFP cells resembled basket cells, with densely branching axons, which have been shown to contact the perisomatic regions of postsynaptic targets (Fig. 4A, top). Physiologically, all of these cells were fast spiking interneurons, easily identified by their narrow spike width and large after hyperpolarization potentials (AHPs). In addition, these cells had high rheobases and fired at high frequencies in response to current injection (Table 1; Fig. $3 A$, bottom). 
A

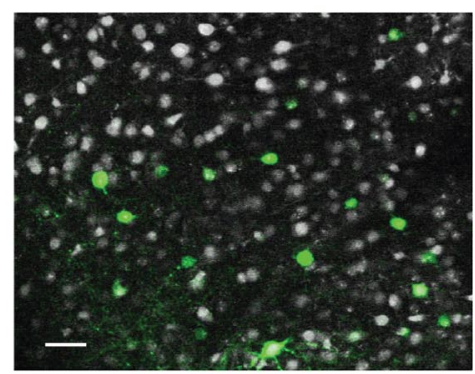

B

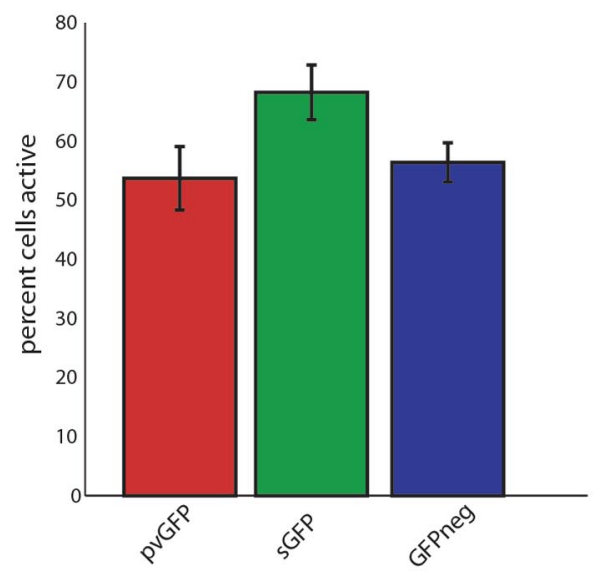

E

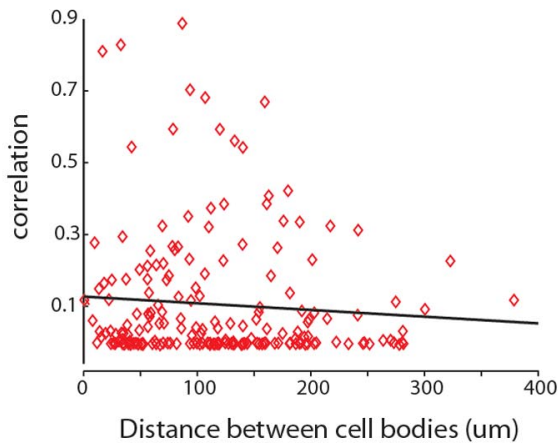

C

raw signals

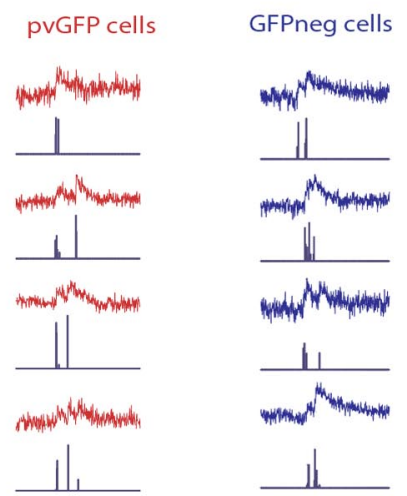

sGFP cells

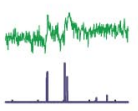

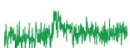

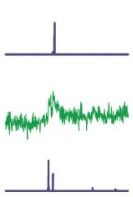

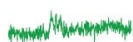

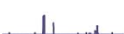

D

spike inference correlation

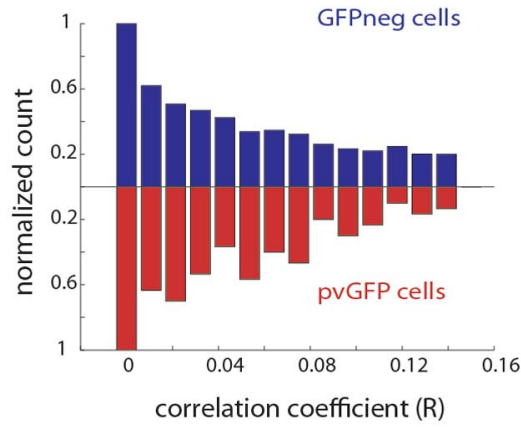

GFPneg cells
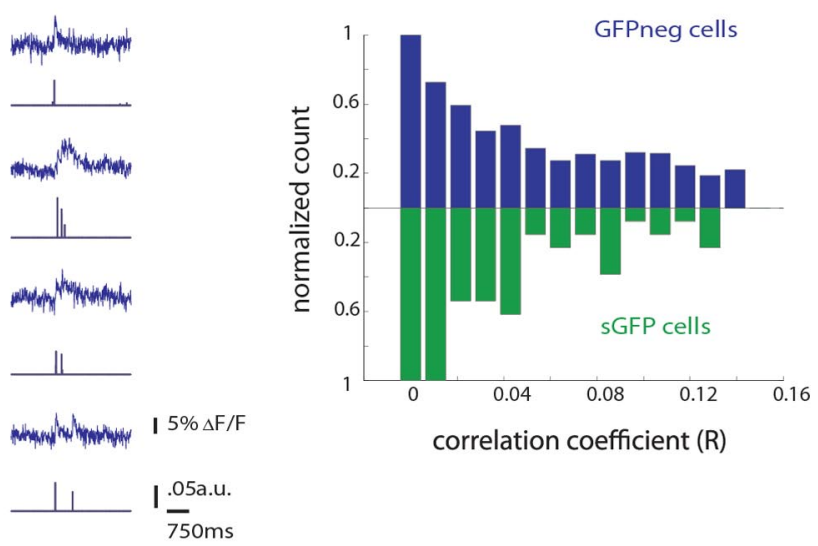
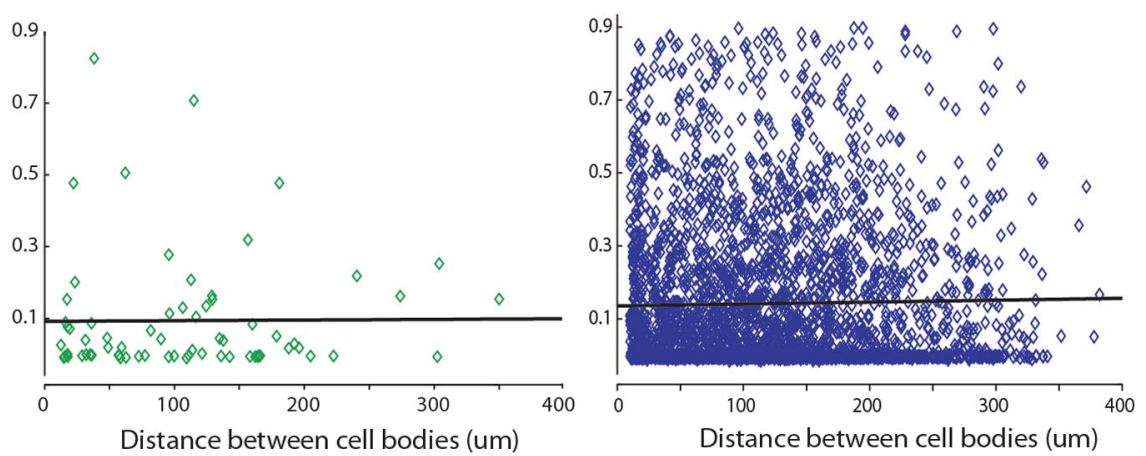

Figure 3. Interneurons are weakly correlated during thalamically triggered activity. $\boldsymbol{A}$, Two-photon image of a loaded slice with labeled interneurons (pvGFP) pseudocolored in green. Scale bar, $30 \mu \mathrm{m}$. B, Quantification of the average percentage active pvGFP (red), SGFP (green), and GFPneg (blue) neurons as determined by SLM imaging and deconvolution. C, Example fluorescence traces from simultaneously imaged pvGFP and GFPneg cells (top) or SGFP cells and GFPneg cells (bottom) during a thalamically triggered UP state. Spike inference for each trace is shown below each example. $\boldsymbol{D}$, Normalized distribution of correlation coefficients of spike inference for pvGFP interneurons and GFPneg cells (top) and sGFP interneurons and GFPneg cells (bottom). These distributions were not significantly different. $\boldsymbol{E}$, Distance versus correlation for all cell pairs imaged. No significant relationship was found between distance and strength of correlation for pvGFP cells (left, $p=$ 0.27 ), sGFP cells (middle, $p=0.78$ ), or GFPneg cells (right, $p=0.27$ ).

We also performed whole-cell electrophysiological recordings from 50 sGFP interneurons. All recorded cells were indeed interneurons, and were characterized both anatomically and physiologically, in a similar manner as pvGFP cells. Anatomically, sGFP cells had typically ascending axon collaterals that branched extensively in layer 1, characteristic of Martinotti cells (Wang et al., 2004; McGarry et al., 2010; Fino and Yuste, 2011) (Fig. 4B, top). Electrophysiologically, in response to current injections, these cells displayed a lower rheobase than fast spiking cells, and a more moderate frequency of discharge, with significant spike frequency adaptation (Table 1; Fig. $4 B$, bottom). A majority of GFPneg cells (62/70 neurons) were confirmed, with a combination of intracellular recordings and anatomical reconstructions, to be excitatory pyramidal and regular spiking neurons, which will refer to as principal cells (PCs) (Fig. 4C).

To address the question of whether nearby interneurons have synchronous firing patterns, we patched pairs of interneurons within $100 \mu \mathrm{m}$ each other in somatosensory cortex layer $2 / 3$, where the probability of both chemical and electrical junctions between these cells is high (Galarreta and Hestrin, 2002). We 
A
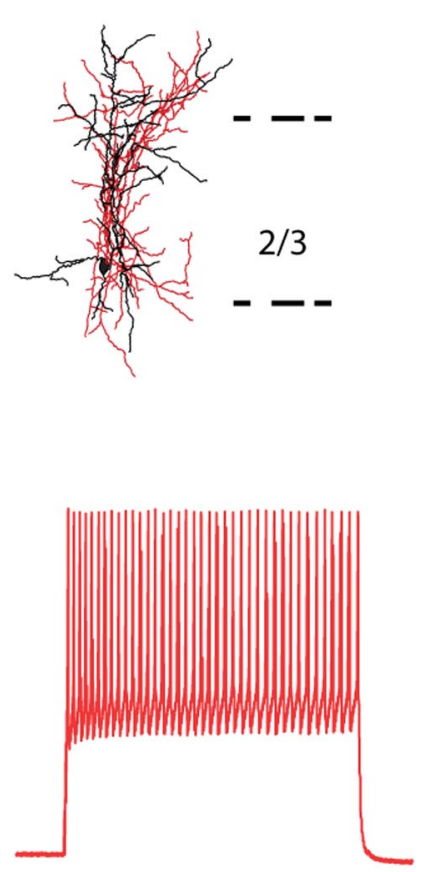

B
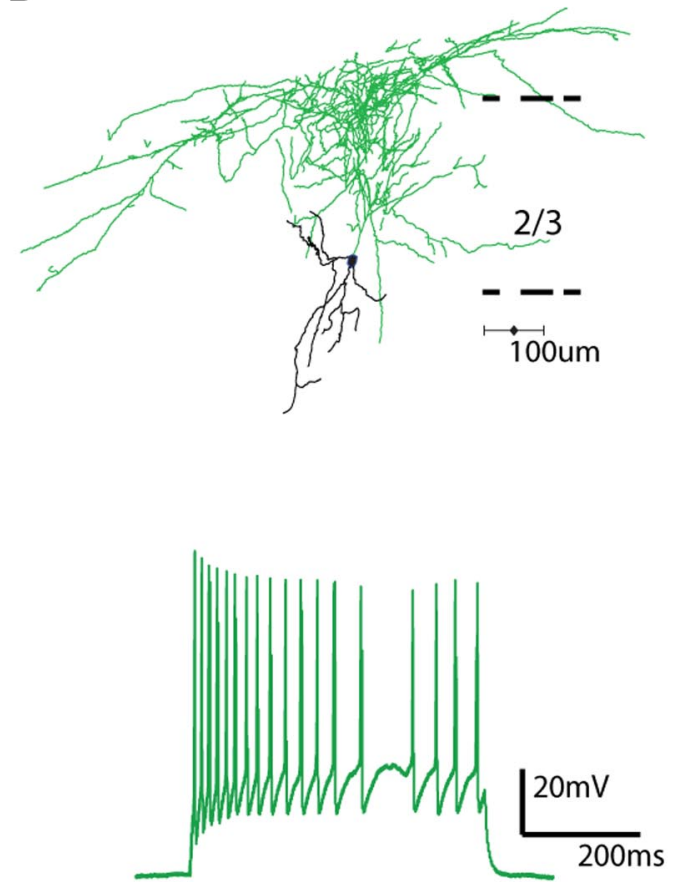

C
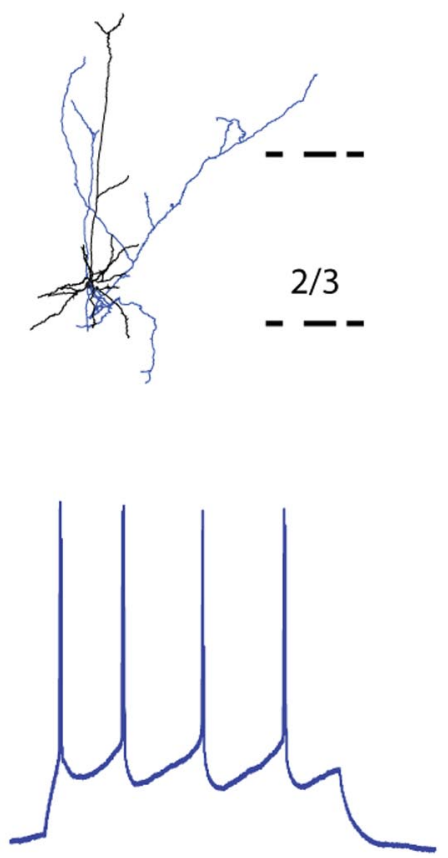

Figure 4. Morphological and physiological characterization of interneurons. A, Reconstruction of a pvGFP-positive basket cell (top), with typical fast spiking physiology (below). Axons in red, dendrites in black. $\boldsymbol{B}$, Reconstruction of a sGFP-positive Martinotti cell, with typical accommodating firing pattern shown below. Axons in green, dendrites in black. C, Reconstruction of a layer $2 / 3$ $\mathrm{PC}$ with characteristic regular firing pattern shown below. Axons in blue, dendrites in black.

Table 1. Electrophysiological properties of pvGFP, sGFP and PC cells

\begin{tabular}{|c|c|c|c|}
\hline & pvGFP & SGFP & $P C$ \\
\hline Number of cells & 42 & 50 & 62 \\
\hline Input resistance $(\mathrm{M} \Delta)$ & $225 \pm 29.0$ & $400 \pm 88.9$ & $542 \pm 76.3$ \\
\hline AP half-width (ms) & $0.712 \pm 0.07$ & $1.05 \pm 0.09$ & $1.65 \pm 0.13$ \\
\hline Resting membrane potential (mV) & $-70.6 \pm 1.27$ & $-67.8 \pm 0.92$ & $-71.5 \pm 0.82$ \\
\hline Rheobase (pA) & $218.6 \pm 31.78$ & $148.4 \pm 25.25$ & $51.1 \pm 9.15$ \\
\hline Firing frequency at $2 \times$ threshold $(\mathrm{Hz})$ & $65.0 \pm 9.04$ & $24.9 \pm 3.14$ & $11.0 \pm 0.82$ \\
\hline Spike frequency Adaptation & $0.79 \pm 0.03$ & $0.41 \pm 0.04$ & $0.72 \pm 0.04$ \\
\hline
\end{tabular}

patched 2-4 cells simultaneously to increase the likelihood of observing pairs in which at least two interneurons fired action potentials in response to thalamic stimulation (Fig. $5 A$ ). In addition, because we could record continuously for long periods of time, we were able to record spontaneously occurring UP states. For both thalamically evoked and spontaneously occurring activity, we calculated the time between spikes in every pair of two active cells. In this way, for each spike, we calculated the shortest time between spikes ("minimum intercell spike interval") for the two cells patched (Fig. 5B). We performed this same analysis for pairs of excitatory PCs firing action potentials in response to thalamic stimulation. After extracting all minimum intercell spike intervals between all pairs of either pvGFP/pvGFP $(n=10$ pairs) or sGFP/sGFP ( $n=8$ pairs) cells or PC/PC cells $(n=15$ pairs) (Fig. $5 C$ ), we constructed probability distributions of minimum time between spikes, which show the likelihood of a minimum intercell spike interval falling within any given $20 \mathrm{~ms}$ time bin, from 0 to $1 \mathrm{~s}$. In performing this analysis, we limited our dataset to experiments in which both neurons fired action potentials in at least three trials (cases in which both neurons did not spike in the same UP state were excluded from the analysis). Surprisingly, the overall minimum spike interval probability distributions were not statistically different between pvGFP, sGFP, and PC cells when compared in a bin-by-bin manner (Fig. 5D) (Friedman test, $p=0.6174$,). This means that spikes do not occur more synchronously in these two interneuron populations than the general population of principal excitatory cells. We also calculated the average minimum time between spikes in all three cell types, and found no significant differences (pvGFP, $225 \pm 18.8$ ms, $n=12$ pairs; sGFP, $191 \pm 15.5 \mathrm{~ms}, n=11$ pairs; PC, $194 \pm$ $9.93 \mathrm{~ms}, n=18$ pairs; $p=0.12$, Kruskal-Wallis test,). Similarly, for spontaneously occurring activity, we found no difference in either the distribution of minimum spike times (Fig. 5E) ( $p=$ 0.25 , Friedman test), or the average minimum spike times (pvGFP, $209 \pm 17.2 \mathrm{~ms}, n=16$; sGFP, $233 \pm 16.1 \mathrm{~ms}, n=6$; PC, $191 \pm 21 \mathrm{~ms}, n=13, p=0.08$, Kruskal-Wallis test). To determine whether or not spiking was more or less synchronous than what would be expected by chance, we reshuffled the spikes, and recalculated the minimum interspike intervals. In all three cells types, PV, SOM, and PC, we found no differences in the average minimum spike time intervals between the experimentally acquired data, and the randomly reshuffled datasets (PV, $247.7 \pm 19.2 \mathrm{~ms}$, $p=0.33$; SOM, $200.6 \pm 14.6 \mathrm{~ms}, p=0.12$; PC, $200.6 \pm 10.0 \mathrm{~ms}, p=$ 0.51 , in all cases Friedman test was used and reshuffled distributions were compared with evoked).

We next limited our analysis to pairs of interneurons coupled electrically. Interneurons were patched within $50-100 \mu \mathrm{m}$ from one another, as shown in Figure 6A. To measure electrical coupling, 500 ms current steps were injected into one neuron or the other, and the coupling coefficient (CC) was defined as the ratio of the voltage deflection in the noninjected cell to the voltage deflection in the injected cell just before current offset, averaged $>10$ sweeps and then averaged in the two directions of connectivity (Fig. 6B). Five of 12 pvGFP and 4 of 11 sGFP pairs were electrically coupled, with coupling coefficients of $0.031 \pm 0.008$ and $0.085 \pm 0.012$, respectively. UP states were then triggered 
A

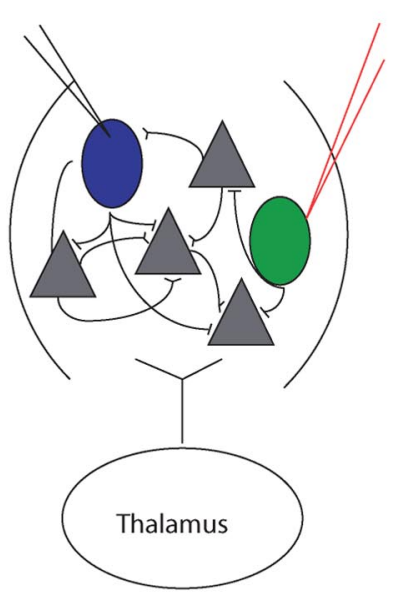

C

pVGFP

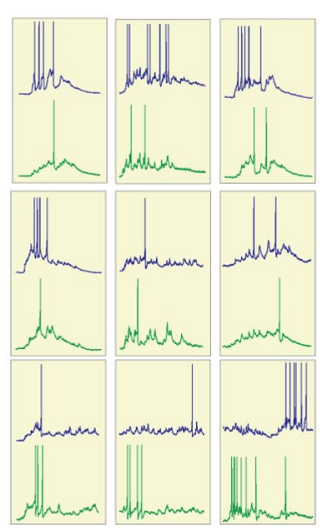

sGFP

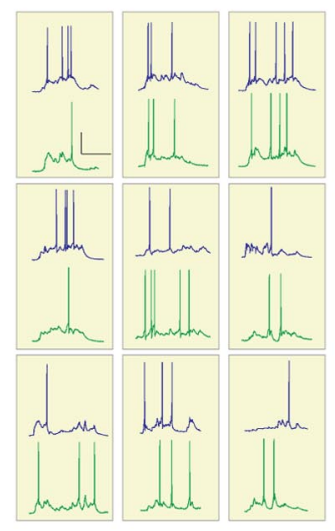

PC

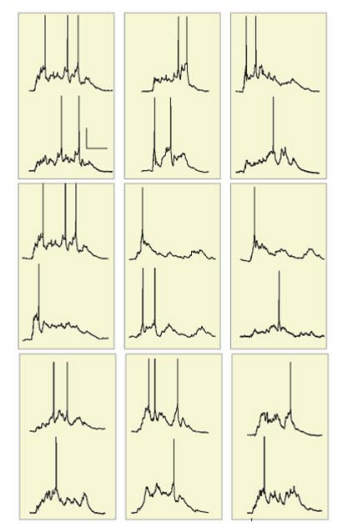

B
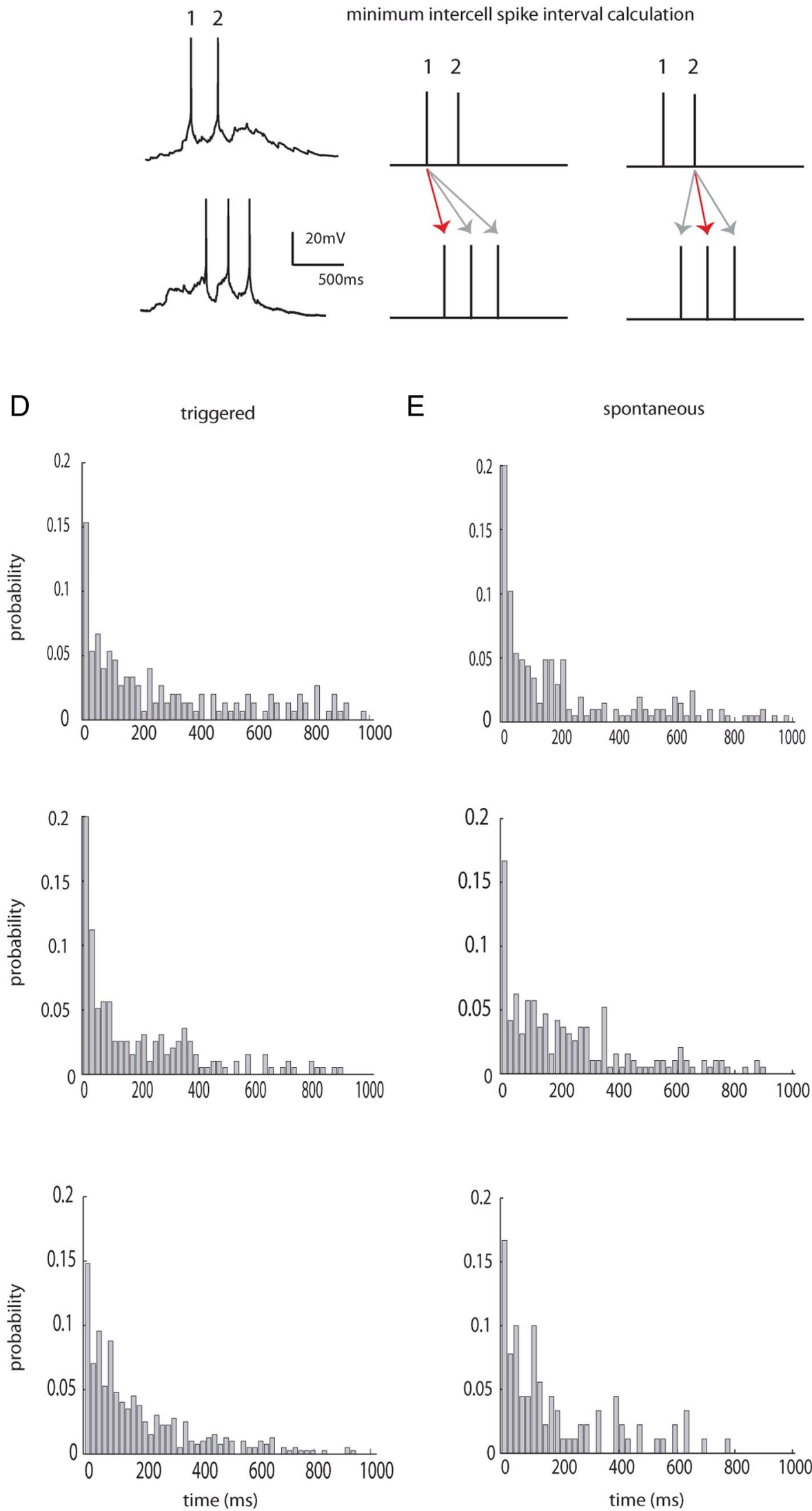

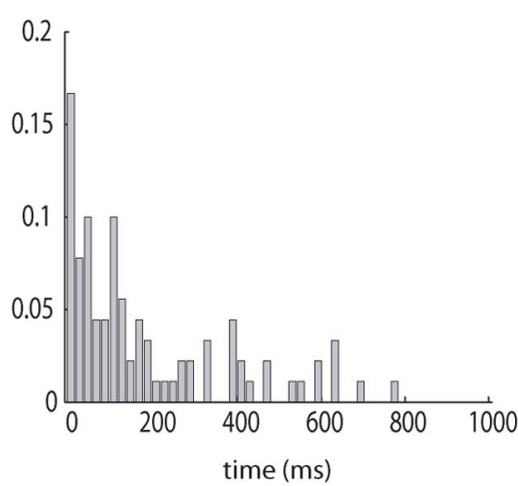

Figure 5. Spiking of interneuron subtypes is not more synchronous than pyramidal cells. $A$, Diagram depicting a layer 4 small recurrent network of cells consisting of interneurons (green and blue) and principal cells (gray). Two nearby cells, either pvGFP, SGFP, or unlabeled PCs were patched in whole-cells current-clamp mode within $200 \mu$ m of one another. $\boldsymbol{B}$, Left, electrophysiological traces from two nearby sGFP cells. Right, a schematic depicting how the minimum intercell spike interval was calculated for each spike in the top trace to the right. (Figure legend continues.) 
with thalamic stimulation, and minimum intercell spike intervals were calculated. We found that the average intercell spike interval of electrically coupled pvGFP cells did not differ from uncoupled ones (Fig. $6 D 1$, left column) $(251.7 \pm 52.4 \mathrm{~ms}$, coupled vs $225 \pm$ $18.8 \mathrm{~ms}$, uncoupled $p=0.29$, Mann-Whitney test). Similarly, for sGFP cell pairs, the average minimum intercell spike intervals was also not significantly different (Fig. 6D2, right column) (183.2 \pm $19.3 \mathrm{~ms}$, coupled vs $191 \pm 15.5 \mathrm{~ms}$, uncoupled; $p=0.38$, MannWhitney test).

Because many changes in plasticity and inhibition occur throughout development, we performed a set of control experiments in young adult (p28-35) animals. Again, we observed no difference when we compared average minimum intercell spike intervals in coupled $(n=3)$ versus uncoupled $(n=4)$ pvGFP cell pairs (Fig. 6D2, left column) $(221 \pm 25.4 \mathrm{~ms}$ coupled vs $238 \pm$ 38.2 uncoupled, $p=0.41$, Mann-Whitney test). This was also the case for electrically coupled $(n=3)$ versus uncoupled sGFP pairs $(n=5)$ (Fig. 6D2, right column) $(214 \pm 29.1$ coupled vs $224 \pm$ 18.5 ms uncoupled; $p=0.37$, Mann-Whitney test).

In the experiments outlined above, the slice exhibited spontaneous UP states, but at low frequencies $(0.005 \mathrm{~Hz} \pm 0.002 \mathrm{~Hz})$. We wondered whether our results were biased by the sparseness of activity, and sought to increase the frequency of the UP states so it would more closely resemble the slow oscillations seen in vivo (Sanchez-Vives and McCormick, 2000; Hasenstaub et al., 2005; Luczak et al., 2007). When slices were bathed in a modified version of ACSF with an increased $\mathrm{Ca}^{2+} / \mathrm{Mg}^{2+}$ ratio (SanchezVives and McCormick, 2000; Xu et al., 2013) and 3.5 mm potassium ("normal $\mathrm{K}^{+}$"), the frequency of the UP states increased significantly (Fig. $7 A$, top trace) $(0.047 \pm 0.009 \mathrm{~Hz}, n=13 ; p<$ $0.001, t$ test). When we increased the potassium concentration to $5 \mathrm{~mm}$ ("high $\mathrm{K}^{+}$"), we found that the frequency of the spontaneously occurring UP states increased even further (Fig. 7A, bottom trace, $B)(0.49 \pm 0.04 \mathrm{~Hz}, n=5 ; p<0.001, t$ test $)$. We then calculated the duration of the UP states, and number of APs fired by each neuron during the UP state, and tested what effect thalamic stimulation would have under such conditions. We found that the duration of each spontaneous UP state ("spont") was significantly longer in normal $\mathrm{K}^{+}(3.33 \pm 0.14 \mathrm{~s})$ versus high $\mathrm{K}^{+}$ $(1.36 \pm 0.19 \mathrm{~s})$. The duration of thalamically stimulated UP states ("stim") was also significantly longer in normal $\mathrm{K}^{+}$versus high $\mathrm{K}^{+}(3.02 \pm 0.18 \mathrm{~s}$ vs $1.53 \pm 0.24 \mathrm{~s})$, and these durations did not differ significantly from spontaneous in each condition (Fig. 7C) $\left(p<0.001\right.$, one-way ANOVA; $p<0.001$ normal $\mathrm{K}^{+}$vs high $\mathrm{K}^{+}$ spont and normal $\mathrm{K}^{+}$vs high $\mathrm{K}^{+}$stim; $p>0.05$ spont vs stim normal $\mathrm{K}^{+}$and spont vs stim high $\mathrm{K}^{+}$; Tukey-Kramer multiplecomparisons test). The number of action potentials fired by each neuron was on average significantly higher in normal $\mathrm{K}^{+}(3.05 \pm$ $0.24)$ versus high $\mathrm{K}^{+}(1.84 \pm 0.39)$. Likewise, the number of action potentials fired during thalamically stimulated UP states was significantly higher in normal $\mathrm{K}^{+}(3.59 \pm 0.27)$ versus high $\mathrm{K}^{+}(2.05 \pm 0.63)$, but again these values did not differ significantly from spontaneous UP states in either $\mathrm{K}^{+}$concentration (Fig. 7D) $\left(p<0.001\right.$, one-way ANOVA; $p<0.01$ normal $\mathrm{K}^{+}$vs

\section{$\leftarrow$}

(Figure legend continued.) C, Representative traces from pairs of simultaneously patched pvGFP (top), SGFP (middle), and PC (bottom) pairs during thalamic stimulation during trials in which both cells fired action potentials. $D$, Probability distributions of minimum intercell spike time intervals for pvGFP cells, sGFP cells and PCs during thalamic stimulation were not significantly different from one another. $\boldsymbol{E}$, Probability distributions of minimum intercell spike time intervals during spontaneously occurring activations were not significantly different from one another. high $\mathrm{K}^{+}$spont and normal $\mathrm{K}^{+}$vs high $\mathrm{K}^{+}$stim; $p>0.05$ spont vs stim normal $\mathrm{K}^{+}$and spont vs stim high $\mathrm{K}^{+}$; Tukey-Kramer multiple-comparisons test). These results are in good agreement with previous work showing that thalamically evoked and spontaneous occurring UP states activate the same subnetworks in cortex (MacLean et al., 2005; Luczak et al., 2007). We now extend this work, showing that even in an oscillating preparation with UP states that occur more frequently but are shorter in duration, thalamic stimulation evokes activations that retain the properties of spontaneous activity.

Using this preparation with higher frequencies of UP states, we investigated whether interneurons would be more or less synchronous than PCs. We again calculated the minimum intercell spike interval among PV cells and PCs within $100 \mu \mathrm{m}$ of one another, and plotted the distributions for both spontaneous and thalamically triggered UP states. For both spontaneously occurring and stimulated UP states, we found no difference in mean minimum interspike interval for PV (168.1 $\pm 16.4 \mathrm{~ms}$ spont; $172.9 \pm 19.7$ stim, $n=5$ pairs $)$ versus PCs $(179.8 \pm 15.9 \mathrm{~ms}$ spont, $181.4 \pm 14.5 ; n=8$ pairs) ( $p=0.32$ stim comparisons, $p=0.52$ spont comparisons; Kruskal-Wallis test). A similar result was found when comparing the probability distributions of minimum interspike intervals in a bin by bin fashion (Fig. $7 E, F$ ) ( $p=0.27$ spont, $p=0.33$ stim; Friedman test). Therefore, at least under the various parameters we tested, electrical coupling does not significantly alter synchronous firing in interneurons.

\section{IPSCs, but not EPSCs, show high correlation during spontaneous and thalamically evoked cortical activations}

Because we found, in both our imaging and electrophysiological studies, that interneurons do not exhibit strong synchrony during thalamically evoked or spontaneous activity, we next investigated the timing of IPSPs compared with EPSPs in nearby PC cells. Such a measurement would be representative of all synaptic inputs and could lend insight into the functional organization of inhibition versus excitation within individual cells. To this end, we used single-electrode voltage-clamp to separate IPSCs from EPSPs. We used an intracellular solution with a chloride reversal of $-70 \mathrm{mV}$, allowing us to isolate EPSCs at this potential, whereas IPSCs were isolated by clamping at $0 \mathrm{mV}$ (Fig. 8A). Two to four PC cells within $200 \mu \mathrm{m}$ within the same layer (2/3) and barrel were patch-clamped, and EPSCs and IPSCs were recorded from these cells on alternate trials, during both triggered and spontaneous activations.

We found that IPSCs showed significantly higher correlations than EPSCs in both thalamically triggered (Figs. $8 \mathrm{~B}, \mathrm{C}$ ) (EPSCs $0.55 \pm 0.04$; IPSCs, $r=0.76 \pm 0.04, n=43$ pairs; $p<0.001$, Mann-Whitney test, $n=43$ pairs) and spontaneous cortical activity (EPSCs $0.46 \pm 0.04$, IPSCs, $r=0.66 \pm 0.04 n=26$ pairs; $p<0.01$, Mann-Whitney test). Analysis of the cross-correlation of EPSCs and IPSCs revealed the half-width at half-height of the cross correlogram was significantly wider for EPSCs than IPSCs (Fig. 8D) (156.2 $\pm 17.1 \mathrm{~ms}$ for EPSCs; $68.2 \pm 7.49$ ms for IPSCs; $p<0.001$, Mann-Whitney Test).

Because IPSCs and EPSCs during UP states have very different rise times (IPSC $3.67 \pm 0.24 \mathrm{~ms}$, EPSC $2.14 \pm 0.17 ; p<0.001$; $n=27$ cells, unpaired $t$ test with Welch correction), decay times (IPSC $17.50 \pm 1.90 \mathrm{~ms}$, EPSC $8.85 \pm 0.45 \mathrm{~ms} ; p<0.001 ; n=27$ cells), and amplitudes (IPSC 77.14 $\pm 8.80 \mathrm{pA}$, EPSC $22.16 \pm 1.51$ $\mathrm{pA} ; p<0.001)$, it is possible that these parameters could affect the values of correlation obtained by correlating the raw traces. More specifically, the longer decay times typically seen in IPSCs could increase the correlation value. To address this issue, we detected 
A

B

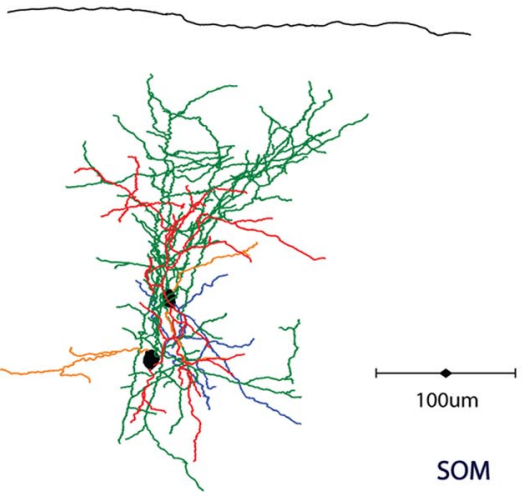

C
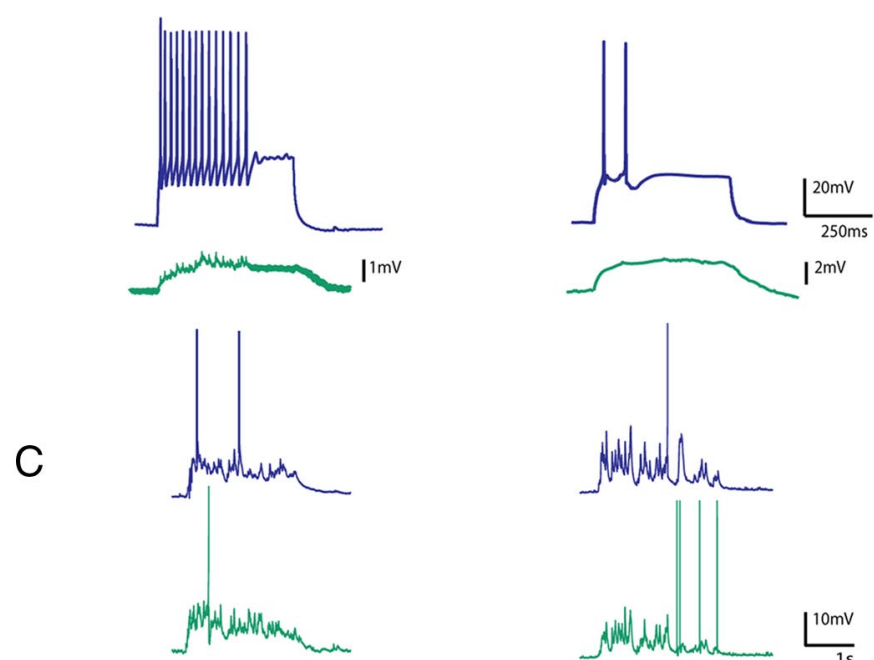

D1

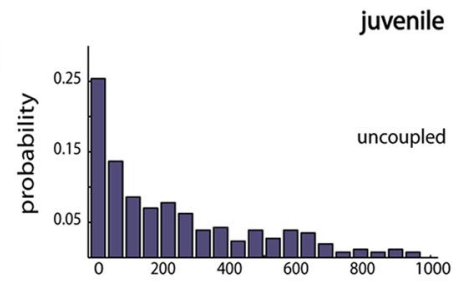

juvenile
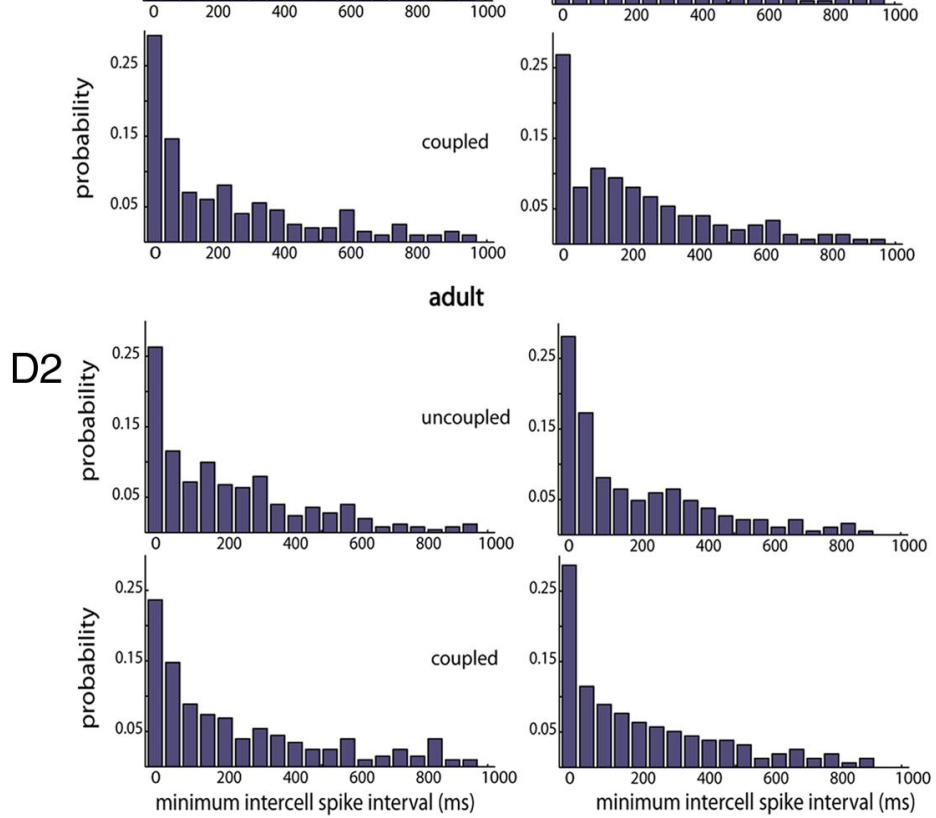

Figure 6. Electrical coupling does not influence synchrony of interneurons. A, Anatomical reconstruction of two pvGFP cells patched within $60 \mu \mathrm{m}$ of one another that were gap junction coupled (axons of cell 1 are in green, dendrites in red, axons cell 2 in blue, dendrites orange). $\boldsymbol{B}$, Intracellular current injections in a PV neuron and a SOM neuron at $2 \times$ rheobase (blue traces top), with

the times of peaks of both EPSCs and IPSCs, converted these into binary time vectors, and correlated these vectors, using time bins of three different sizes $(1,10$, and 100 milliseconds). In this way, instead of correlating the events themselves, we correlated only the timing of the events. But even at the smallest time bins analyzed ( $1 \mathrm{~ms}$ ), the time vectors of IPSCs were significantly more correlated than EPSC time vectors (Fig. 9A) (EPSCs, $r=$ $0.037 \pm 0.02$; IPSCs, $r=0.090 \pm 0.01 ; p<$ 0.001 , unpaired $t$ test, $n=27$ pairs). This was also true at larger time bins we checked, $10 \mathrm{~ms}$ (Fig. 9B) (EPSCs, $r=$ $0.181 \pm 0.02$; IPSCs, $r=0.454 \pm 0.01$; unpaired $t$ test, $p<0.001$ ), and $100 \mathrm{~ms}$ (Fig. 9C) (EPSCs, $r=0.360 \pm 0.025$; IPSCs, $r=0.695 \pm 0.027$; unpaired $t$ test, $p<0.001$ ), in both spontaneously occurring and triggered cortical activity. These results indicated that inhibitory potentials are significantly more correlated than excitatory ones, a finding apparently at odds with our previously found lack of difference in the correlation of firing of excitatory and inhibitory cells.

\section{Common inhibitory input underlies correlated IPSCs}

Two different scenarios could explain the synchronous IPSCs we found during triggered and spontaneous activity. First, it is possible that interneurons are firing more synchronously than their excitatory counterparts, and such synchronization would lead to IPSCs occurring at the same time in nearby pyramidal cells (Fig. 10A, left). Although this could explain the high degree of IPSC correlations observed, our population imaging and dual intracellular recordings failed to show any significant difference in the correlations between pyramidal cells and interneurons. A second possibility is that the higher correlation of IPSCs could be due to shared presynaptic input, emanating from the same neuron. In this case, an interneuron would have to be densely connected to downstream pyramidal cells within the distances we tested $(30-200 \mu \mathrm{m})$. If this were true, each time an inhibitory interneuron fired an action potential, it would generate a

\footnotetext{
a nearby electrically coupled neuron patched within $100 \mu \mathrm{m}$ (green traces, bottom). C, Representative traces from a pair of PV interneurons, and SOM interneurons, respectively, in response to thalamic stimulation. $\boldsymbol{D}$, Distribution of minimum spike times in PV (left) and SOM (right) interneurons in juvenile (D1) and adult animals (D2). Mean minimum intercell spike time distributions did not differ between uncoupled and coupled neurons in either juvenile or adult slices.
} 
A

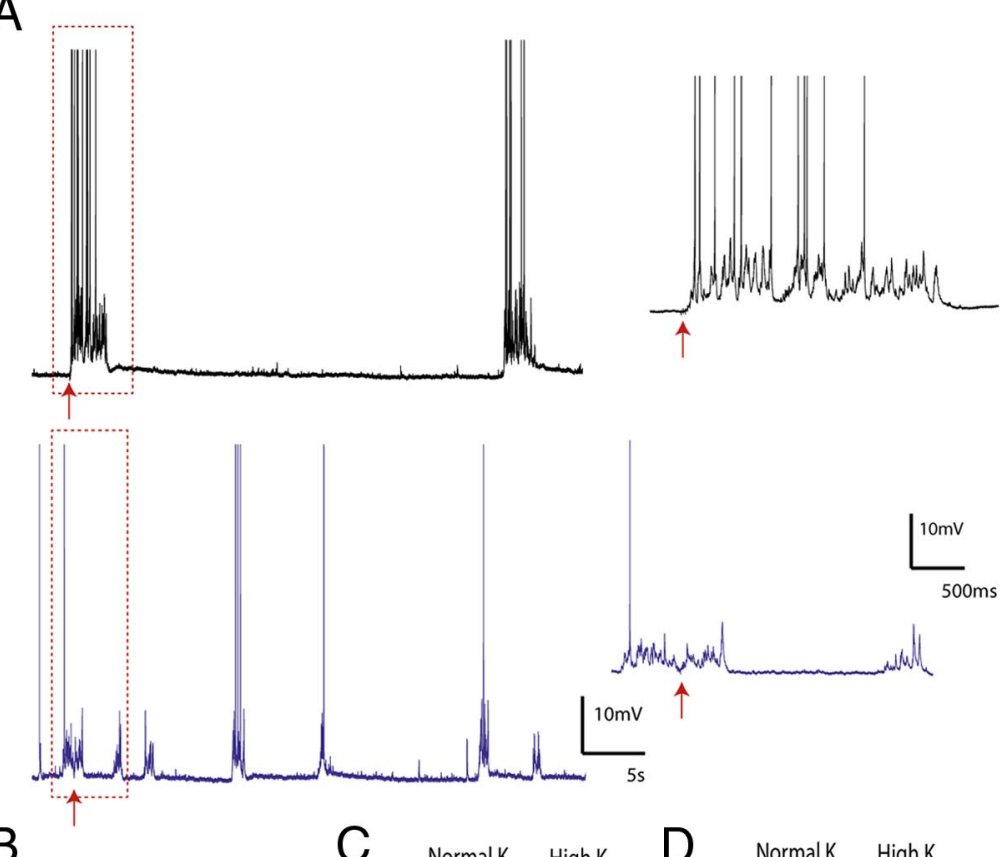

B

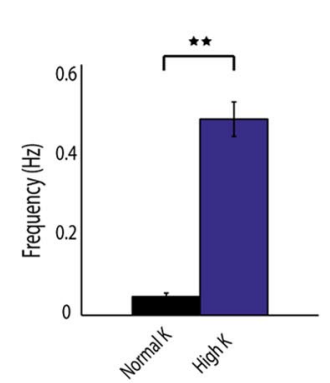

$E$

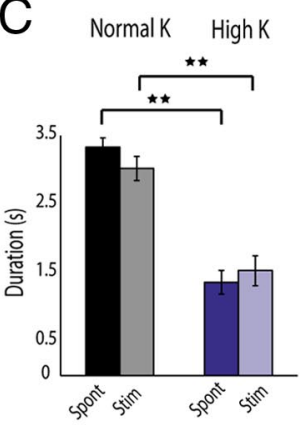

Spontaneous
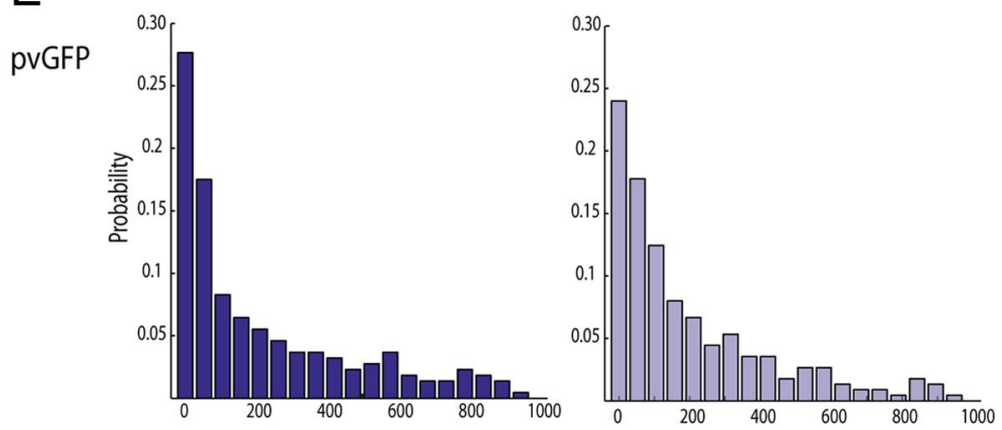

$\mathrm{F}$

PC
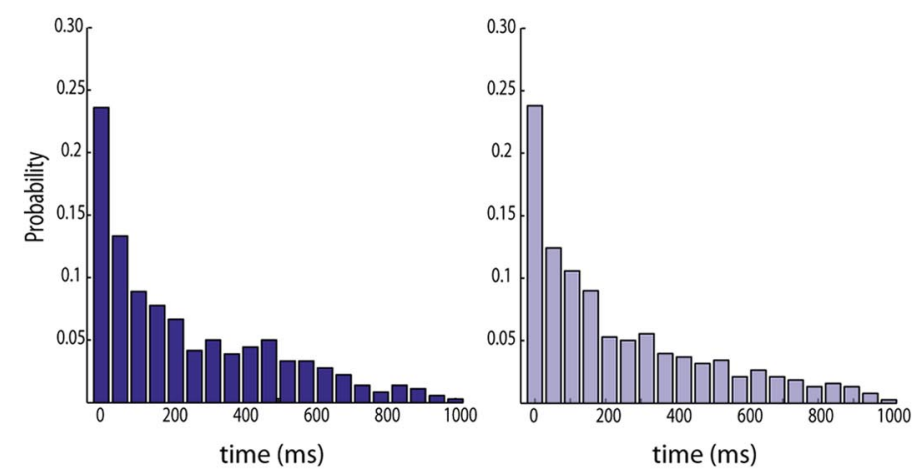

Figure 7. Interneurons are not synchronous during low-frequency oscillations. $A$, Thalamic activation of cortical neurons in two different UP states regimes. Recordings were made from pvGFP interneurons and PCs in ACSF with either normal $\mathrm{K}^{+}$(top trace, black) or high $\mathrm{K}^{+}$(bottom trace, purple). The thalamus was stimulated in both of these conditions, and the response to this

synchronous IPSC in its downstream targets, thus explaining the emergence of correlated IPSCs (Fig. 10A, right).

Because our imaging and electrophysiological experiments led us to conclude that only a minority of the high correlation we observed in IPSCs could be due to synchronous firing, we hypothesized that a high degree of overlapping input from nearby interneurons was primarily responsible for the correlated IPSCs. If correlation of IPSCs is due to shared input rather than synchronous firing, two criteria would need to be met: (1) inhibitory connections onto PCs should be much more dense locally than connections from PCs to PCs and (2) each IPSC detected during the thalamic response should be attributable to just one or a few interneurons. To investigate this, we performed paired recordings between either pvGFP cells and PCs, or SOM cells and PCs. We found that, within $150 \mu \mathrm{m}, 79.8 \pm 12.0 \%$ of dually patched pvGFP and PC cell pairs were monosynaptically connected. We found a similarly high connection probability between sGFP cells and PC cell pairs $(74.8 \pm 11.0 \%)$, whereas the connection probability between PC pairs was significantly lower $9.8 \pm 3.1 \%$; (Fig. 10B) $(p=$ 0.001 , Kruskal-Wallis; $p<0.01$ for PC vs pvGFP and PC vs sGFP; $p>0.05$ for pvGFP vs sGFP, Dunn's multiple-comparison test). This high probability of connection of interneurons onto PCs fulfills the first criteria, and is consistent with recent results demonstrating a dense connectivity from somatostatin and parvalbuminpositive interneurons to neighboring pyramidal cells (Fino and Yuste, 2011; Packer and Yuste, 2011). We also examined how correlations of IPSCs and EPSCs drop off with distance, because connection probability of both $\mathrm{PV} \rightarrow \mathrm{PC}$ and $\mathrm{SOM} \rightarrow \mathrm{PC}$ has

\section{$\leftarrow$}

stimulation is shown in the expanded traces to the right. $\boldsymbol{B}$, The frequency of UP states in high $\mathrm{K}^{+}$was significantly higher than normal $\mathrm{K}^{+}$. C, the duration of the UP state was significantly shorter in high $\mathrm{K}^{+}$versus normal $\mathrm{K}^{+}$for both spont and thalamically stim UP states. No significant difference was found when comparing spont versus stim in either high $\mathrm{K}^{+}$or normal $K^{+} . \boldsymbol{D}$, the number of action potentials fired by PV cells and $P C$ s during the UP state was significantly lower in high $\mathrm{K}^{+}$versus normal $\mathrm{K}^{+}$for both spont and stim UP states. No significant difference was found when comparing spont versus stim in either high $\mathrm{K}^{+}$or normal $\mathrm{K}^{+} .{ }^{* *} p<0.001$, ${ }^{*} p<0.01$. $E$, Distributions of minimum intercell spike intervals for pvGFP interneurons in high $\mathrm{K}^{+}$for both spont (left) and stim (right) conditions. $F$, Distributions of minimum intercell spike intervals for $\mathrm{PC}$ in high $\mathrm{K}^{+}$for both spont (left) and stim (right) conditions. No significant difference was found when comparing pvGFP versus $P C$ distributions in spont and stim conditions. 
A EPSCS
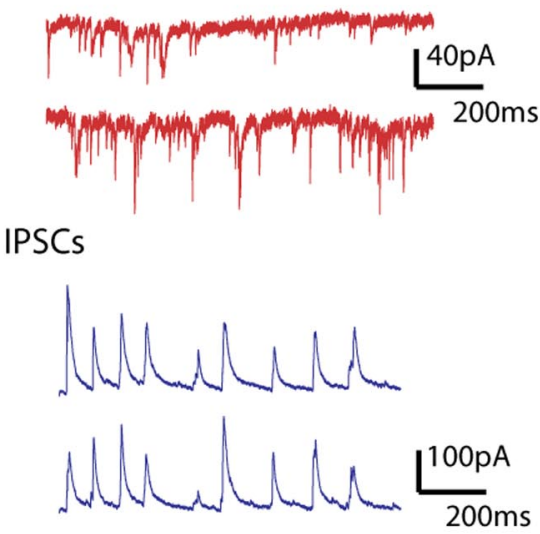

C

Evoked

Correlation (R):

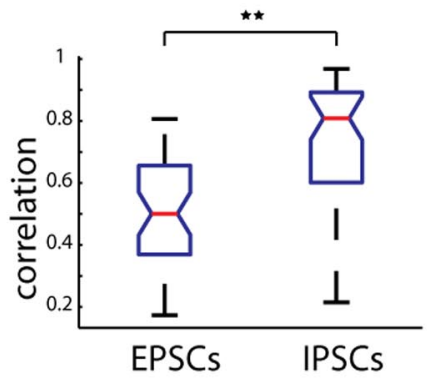

D

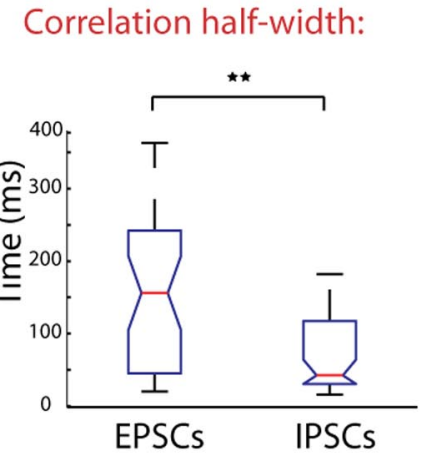

Spontaneous

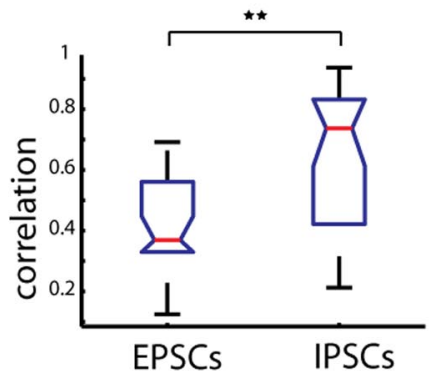

B

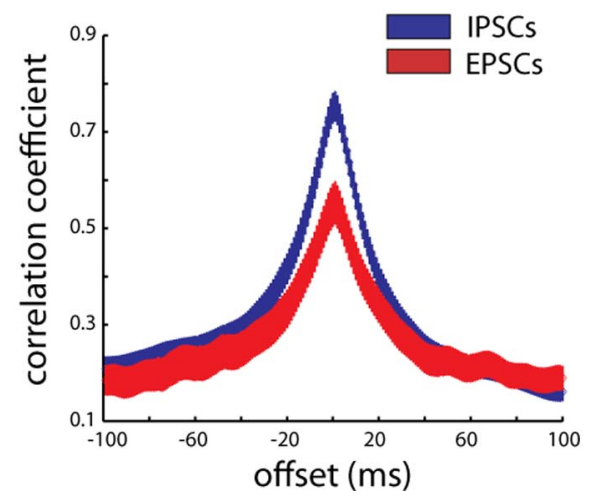

Figure 8. IPSCs are more highly correlated than EPSCs during triggered and spontaneous activations. A, Recordings from two PCs with cell bodies $\sim 68 \mu \mathrm{m}$ apart. Top trace shows EPSCs during thalamic stimulation, bottom shows IPSCs recorded in the same cells on an alternate trial. B, Cross-correlations between currents at $0 \mathrm{mV}$ (blue) and $-70 \mathrm{mV}$ (red). C, Box plots of all correlation coefficients calculated for EPSCs recorded at $-70 \mathrm{mV}$ and IPSCs recorded at $0 \mathrm{mV}$ during evoked activity (left) and spontaneous activity (right). D, Half-width of the cross-correlations for EPSCs and IPSCS. ${ }^{* *} p<0.001$.

been shown to drop off steeply with distance (Fino and Yuste, 2011; Packer and Yuste, 2011). Our rationale was that if synchronization was causing the high correlations of IPSCs, the correlations we observed may remain higher over larger distances than would be expected if they were caused by common input. In accordance with this, both IPSCs and EPSCs dropped off rapidly with distance, with slopes significantly different from zero (Fig. 10C) (EPSCs, $n=30$ pairs, $r_{\text {corr }}=-0.67, p<0.001$; IPSCs, $n=25$ pairs, $r=-0.72$; $p<$ 0.001 ; linear regression) with no difference in their slopes (analysis of covariance $p=0.52$ ).

Next, to estimate of how many interneurons contribute to each IPSC during cortical activity, we measured the conductance of IPSCs during triggered activity, and compared these to the conductance of monosynaptic pvGFP $\rightarrow$ PC and $s G F P \rightarrow P C$ IPSCs, as measured from paired recordings. The mean conductance of pvGFP $\rightarrow$ PC connections was significantly higher than that of $\mathrm{sGFP} \rightarrow \mathrm{PC}$ connections (pvGFP $\rightarrow \mathrm{PC}, 2.08 \pm 0.50 \mathrm{nS}$; $\mathrm{sGFP} \rightarrow \mathrm{PC}, 0.76 \pm 0.24 \mathrm{nS}, t$ test, $p<0.05, n=10$ for $\mathrm{pGFP} \rightarrow$ PC pairs and $n=15 \mathrm{sGFP} \rightarrow$ PC pairs), which is unsurprising given our recordings were made at the soma, much nearer to where parvalbumin interneurons form synapses onto PCs. More importantly, the mean conductances during cortical activity ( $1.11 \pm 0.02 \mathrm{nS}, n=6578$ IPSCS recorded from 15 cells) did not differ significantly from the average conductances of monosynaptic, single axon, PV and SOM inputs $(1.38 \pm 0.29 \mathrm{nS} ; p=$
0.442, Mann-Whitney, $n=25$, normalized distributions) (Fig. $10 D)$. In addition, the failure rate of IPSCs was extremely low (nearly $0 \%$, data not shown), which would ensure that given a dense connectivity scheme, as each time an interneuron fired an action potential it would be recorded in all its downstream targets. Finally, as illustrated in Table 2, the rise time, decay, amplitude and half-width of IPSCs measured from paired recordings did not significantly differ from IPSCs recorded during UP states ( $p<0.001$ for all comparisons, Student's $t$ test). This indicates that each IPSC observed during triggered or spontaneous activity could be generated by just one or at most a few interneurons, fulfilling the second criteria discussed above. We conclude that high correlation of IPSCs seen during thalamically driven UP states must be primarily due to shared presynaptic input from inhibitory neurons rather than synchronous firing of interneurons.

\section{Decreasing inhibition correlates neural activity}

With inhibition itself being asynchronous, we wondered whether a functional role of inhibition could be to "desynchronize", rather than synchronize, the local network. In fact, several network models have proposed that a dynamic balance of excitatory and inhibitory fluctuations counteracts correlations induced by common inputs (Hertz, 2010; Renart et al., 2010). However, direct experimental evidence in support of these models has been 
Evoked

A

A $1 \mathrm{~ms}$

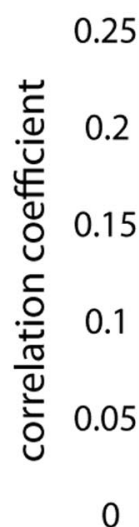

B
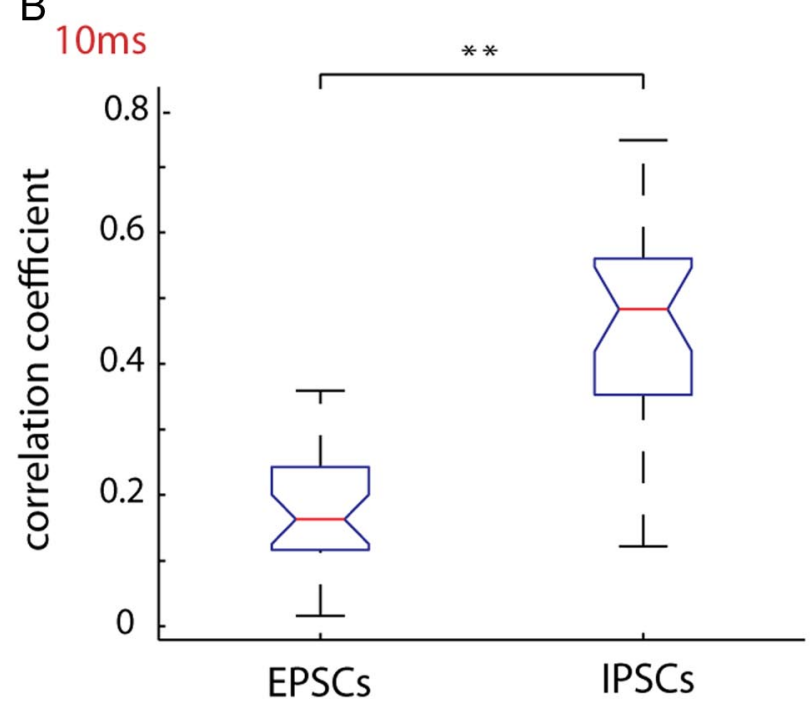

$C_{100 \mathrm{~ms}}$

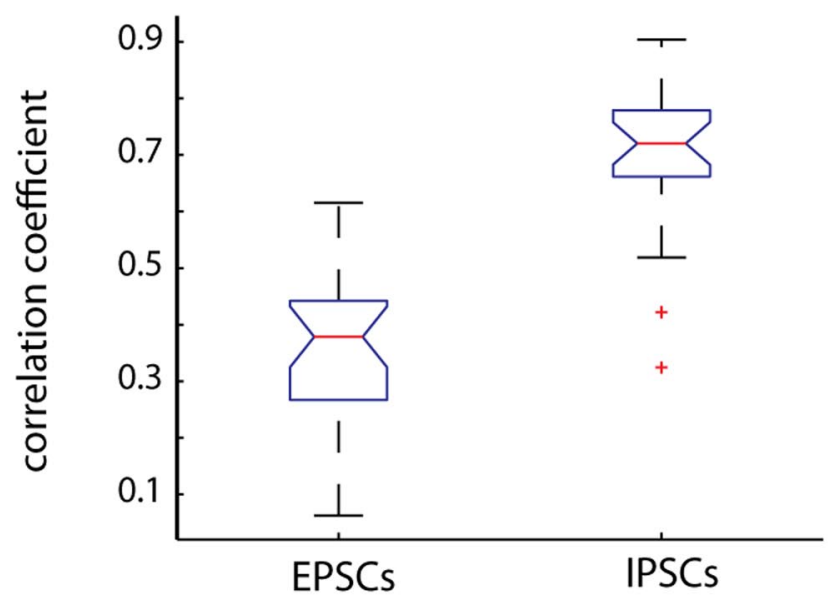

Spontaneous
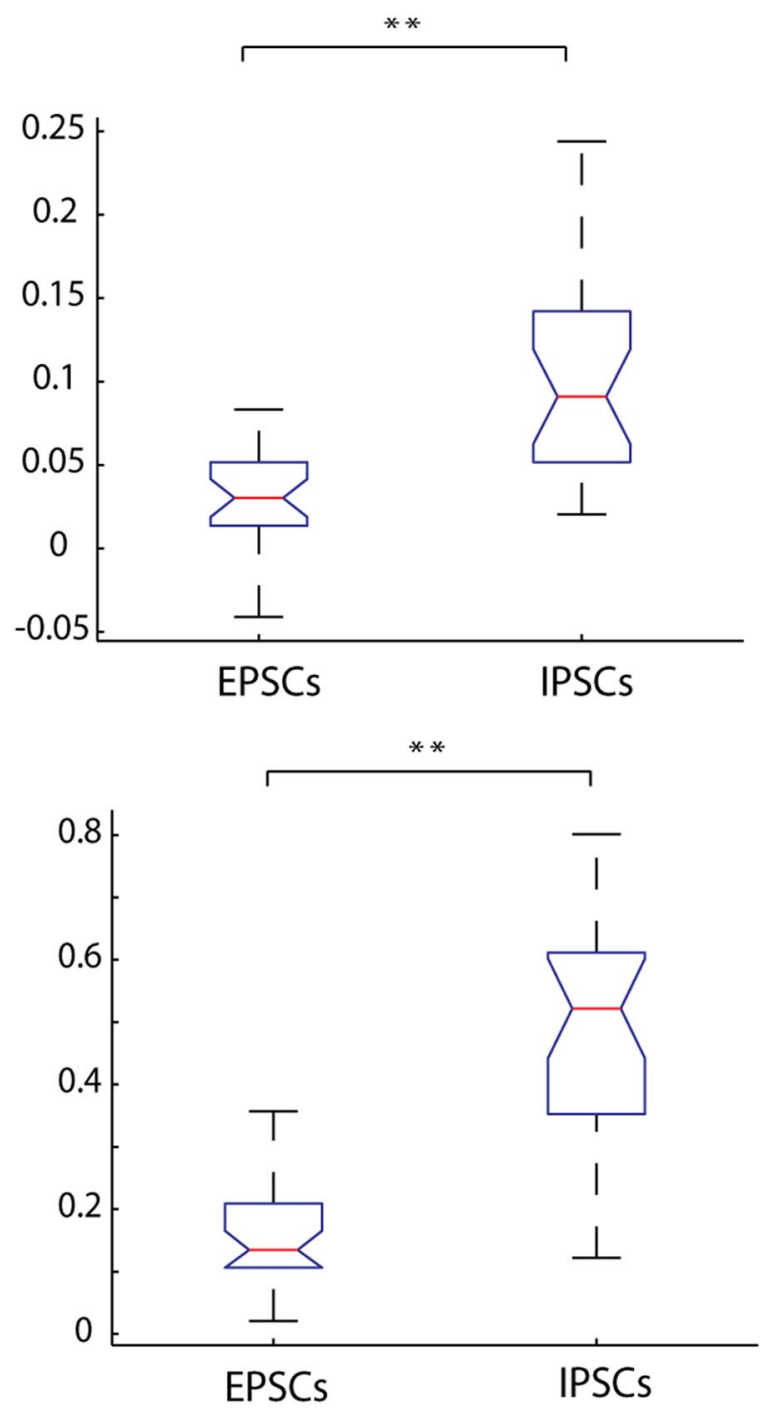

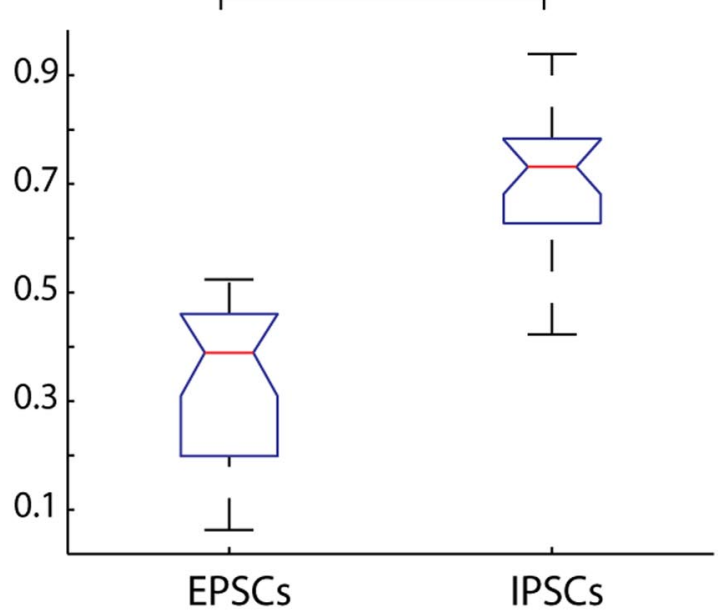

Figure 9. Correlation of unitary EPSCS and IPSCs confirms IPSCS are more synchronous that EPSCs. Individual IPSC 5 and EPSCs were detected and binary vectors of the event times were correlated among simultaneously patched cells pairs. These vectors were binned at either $\boldsymbol{A}, 1 \mathrm{~ms} ; \boldsymbol{B}, 10 \mathrm{~ms}$; $\operatorname{or} \boldsymbol{C}, 100 \mathrm{~ms}$. For all time bins, the correlation coefficient for IPSC was significantly higher than EPSCs for both evoked and spontaneous activity. ${ }^{* *} p<0.001$. 
A synchronous firing
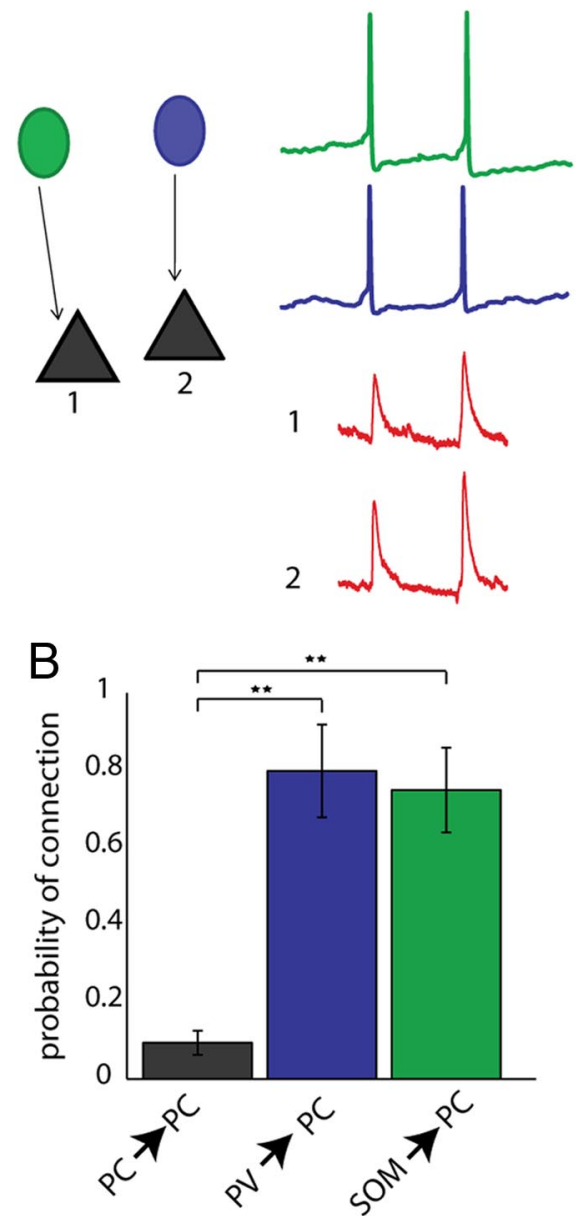

.

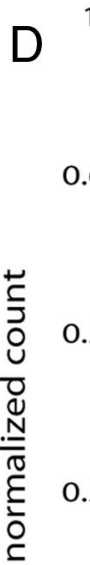

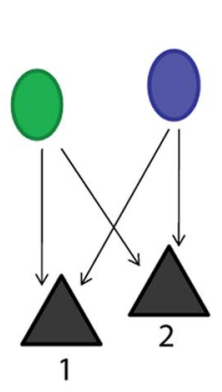

shared input
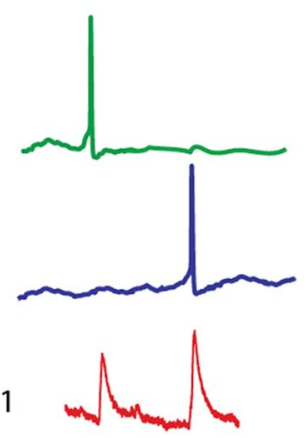

2

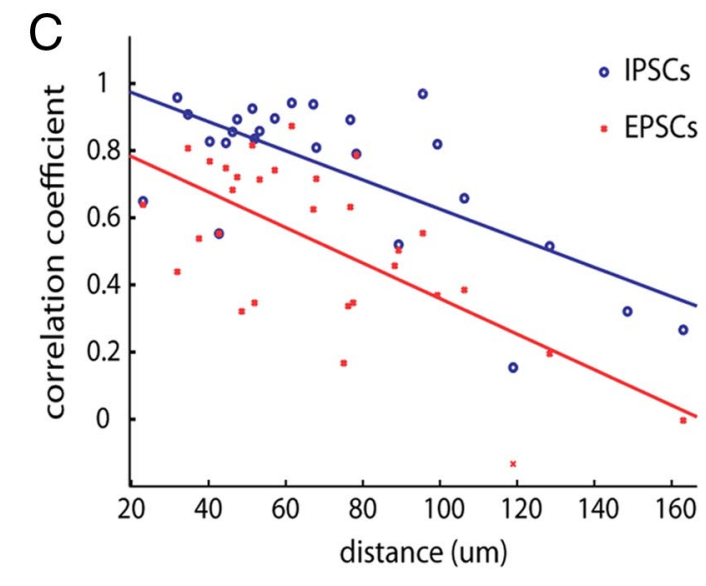

IPSCs thalamic response

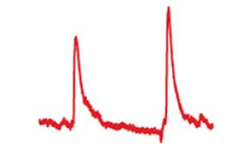

IPSCs connected pairs

1

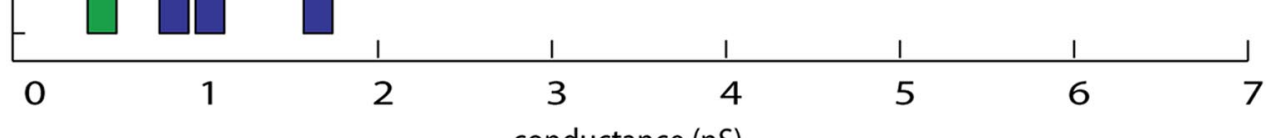

Figure 10. High correlation of IPSCs is due to common input, rather than synchronous firing of interneurons. A, Schematic depicting two possible mechanisms underlying correlated IPSCS. In the first scenario, depicted to the left, synchronous firing, correlated IPSCs would be caused by two or more interneurons firing simultaneously. In this case, each IPSC would (Figure legend continues.) 
Table 2. Similar properties of IPSCs for monosynaptic connections and UP states

\begin{tabular}{lccc}
\hline & IPSC pairs $(n=25)$ & IPSCs UP state $(n=6578)$ & Statistics \\
\hline 10-90 Rise time (ms) & $3.11 \pm 0.37$ & $3.67 \pm 0.24$ & NS \\
Amplitude (pA) & $97.53 \pm 21.06$ & $77.14 \pm 8.81$ & NS \\
Half-width (ms) & $15.98 \pm 0.98$ & $13.76 \pm 0.84$ & NS \\
Decay constant (ms) & $19.10 \pm 1.45$ & $17.50 \pm 1.94$ & NS \\
\hline
\end{tabular}

scant. In their theoretical model of decorrelated networks, Renart et al. (2010) proposed that although isolated EPSCs and IPSCs can be correlated due to common input, these correlations cancel one another, and therefore fall off at intermediate membrane potentials. To test the validity of this model, we again patched 2-4 neurons within $100 \mu \mathrm{m}$ of one another in voltage-clamp during thalamic UP states. We then recorded correlations in membrane potential at several voltages from -70 to $0 \mathrm{mV}$ (Fig. $11 \mathrm{~A}$ ). Interestingly, our experimental data were similar to what was predicted by the model in that the correlation coefficients were highest at the extremes of voltages $(-70 \mathrm{mV}$ and $0 \mathrm{mV})$ (Fig. $11 B$ ) ( $n=18$ pairs), where either mostly EPSCs or IPSCs, respectively, were recorded. Importantly, at intermediate voltages $(n=8$ pairs), the correlation coefficient drops off. This is attributed to the fact that when EPSCs and IPSCs are themselves correlated, as is the case during both spontaneous and sensory-evoked activity in somatosensory cortex (Okun and Lampl, 2008) the correlations in EPSCs and IPSCs occur simultaneously and cancel, leading to a significant reduction in the correlation of membrane potential. A natural consequence of this is that the correlation of output spikes in this scenario will also be low, as we have shown here is the case during UP states.

In a final set of experiments, we hypothesized that if indeed inhibitory currents decorrelated excitatory ones, reducing inhibitory currents should increase membrane potential correlations. To test this, we reduced inhibition with nanomolar (100-200 $\mathrm{nm}$ ) amounts of GZ (SR-95531), and reassessed the correlation coefficients of excitatory currents among two cells within $100 \mu \mathrm{m}$ of one another (Fig. 11C). At these concentrations of GZ, while there is mild reduction of inhibitory currents, epilepsy is not observed (Sanchez-Vives et al., 2010). We found that the pairwise correlation coefficients of EPSCs significantly increased with 100 $\mathrm{nm}$ GZ in the bath, and further increased at $200 \mathrm{~nm}$ of GZ (Fig. $11 D$ ) (control, $r=0.31 \pm 0.04 ; 100 \mathrm{~nm} \mathrm{GZ,} r=0.58 \pm 0.05 ; 200$ $\mathrm{nm} \mathrm{GZ}, r=0.66 \pm 0.05 ; p<0.05$ control vs $100 \mathrm{~nm}$ and control vs $200 \mathrm{~nm}$ Mann-Whitney, $n=5$ pairs). Because disinhibition increases firing rates which may spuriously increase correlations, we sought to determine what effect blocking inhibition had on correlations of reshuffled data. The correlations of reshuffled data did not differ significantly in either $100 \mathrm{~nm}$ or $200 \mathrm{~nm}$ of the drug (control, $r=0.04 \pm 0.02 ; 100 \mathrm{~nm} \mathrm{GZ,} r=0.07 \pm 0.03 ; 200$

\section{$\leftarrow$}

(Figure legend continued.) be the sum of the spiking of several interneurons. In the second scenario, shared input, in a system where every interneuron has highly divergent axons and contacts many postsynaptic PCs, each time an interneuron fires a spike, an IPSC would be recorded from all of its downstream postsynaptic targets nearly simultaneously. $\boldsymbol{B}$, Connection probabilities for $\mathrm{PC} \rightarrow \mathrm{PC}$ pairs pvGFP $\rightarrow \mathrm{PC}$ pairs and $\mathrm{SGFP} \rightarrow \mathrm{PC}$ pairs showing significantly higher connection probability for interneuron $\rightarrow P C$ than for $P C \rightarrow P C(p=0.001$ KruskalWallis; $p<0.001$ for PC vs pvGFP and PC vs sGFP; $p>0.05$ for pvGFP vs sGFP, Dunn's multiplecomparison test; $\left.{ }^{* *} p<0.01\right)$. C, Distance versus correlations coefficients of IPSCs (blue circles) and EPSCs (red squares) were plotted for all cell pairs. Both EPSC and IPSC correlations drop off with distance with slopes that were not significantly different from one another. $\boldsymbol{D}$, Normalized distribution of conductances for IPSC recorded during thalamically triggered activations (top, red), and synaptic conductances measured from pvGFP $\rightarrow P C$ pairs (blue) or sGFP $\rightarrow P C$ pairs (green). The mean of these distributions did not differ from one another. $\mathrm{nm} \mathrm{GZ,} r=0.16 \pm 0.05 ; p=0.90$ for control vs $100 \mathrm{~nm}, p=0.34$ for control vs $200 \mathrm{~nm}, n=5$ pairs). We therefore conclude that there is a causal link between the presence of inhibition and the decorrelated firing of excitatory cells, and that interneurons may be acting to decrease rather than increase correlated activity.

\section{Discussion}

We used fast two-photon calcium imaging and electrophysiology to study the correlations in neuronal spiking activity within three major subclasses: PV and SOM-expressing interneurons, and pyramidal cells. Interneurons exhibited low correlations in response to both thalamic stimulation and during spontaneously occurring UP states. This uncorrelated activity was a general feature of all inhibitory cells; even interneurons within a subclass did not exhibit correlated firing, as compared with pyramidal cells. Intracellular recordings confirmed that the distribution of minimum intercell spike intervals was not significantly different among interneuronal subtypes and/or excitatory cells. Surprisingly, even cell pairs connected by gap junctions did not display tight synchrony as previously reported (Tamas et al., 2000; Deans et al., 2001; Galarreta and Hestrin, 2001; Di Garbo et al., 2005). On the other hand, in apparent contradiction with our findings of uncorrelated activity of interneurons, voltage-clamp recordings demonstrated IPSCs are indeed correlated at close distances, but we found that this phenomenon is due mostly to shared input rather than synchronous firing. Our data thus indicate that, during cortical UP states, asynchronous activity is a general feature of all cortical cells, regardless of cell class. Because UP states represent recurrent activity within local circuits, this uncorrelated activity may serve to provide a backdrop upon which sensory discriminations can be more easily be decoded (Abbott and Dayan, 1999).

\section{Cortical interneurons have uncorrelated firing during UP states}

Although it is clear that there are many subtypes of GABAergic interneurons (Ascoli et al., 2008), we know little about whether different subtypes respond differently to stimuli. Improvements in the ability to identify interneurons has allowed for the assessment of correlations among these cells during sensory driven and spontaneous behavior (Hofer et al., 2011). Although this study was highly informative, the authors addressed the issue of synchrony at a time scale of hundreds of milliseconds, which has fundamentally different consequences on information processing. Our findings, using high speed imaging, demonstrate that subtype specificity does not confer correlated spiking activity in response to thalamically driven or spontaneously occurring UP states. In addition, we find that nearby neurons do not have more correlated activity than those at further distances, at least within the relatively close distances examined.

A drawback of our imaging experiments is that, although PV and SOM neurons were selectively labeled, there are many further subdivisions that can be made. PV interneurons, especially, are thought to represent a number of subtypes including, but not limited to, basket cells, chandelier cells, and multipolar bursting interneurons (Blatow et al., 2003; Wang et al., 2004). Likewise, SOM cells belong to at least three distinct subclasses (McGarry et al., 2010). Therefore one possible interpretation of our imaging data are that they simply did not afford the resolution necessary to detect synchrony among interneurons of the same subclass, within the PV and SOM labeled subtypes.

Our data showing uncorrelated activity among interneurons are surprising given that interneurons are often coupled both electrically 
A

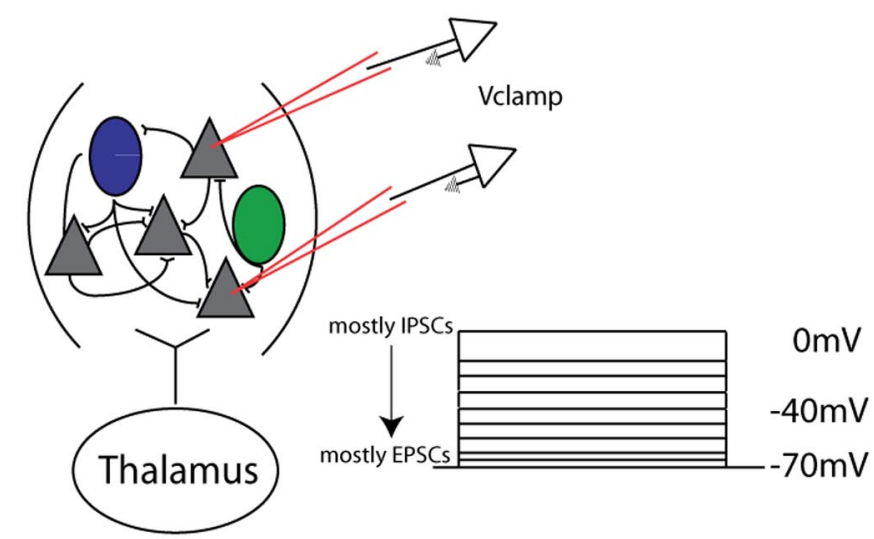

C

control

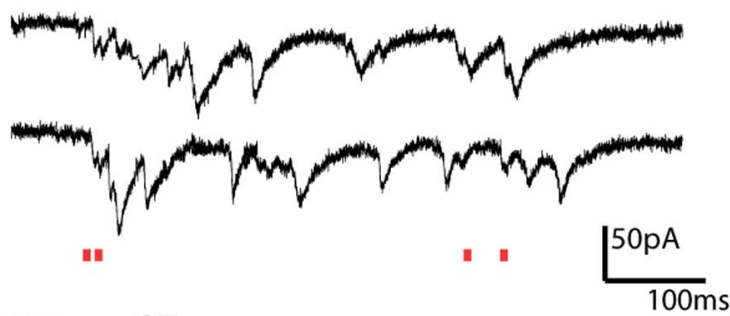

$200 \mathrm{~nm} \mathrm{GZ}$

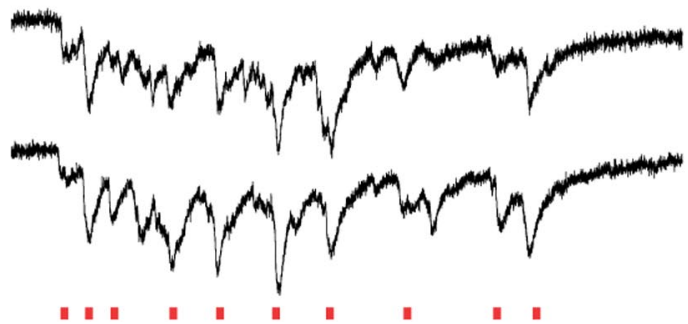

B

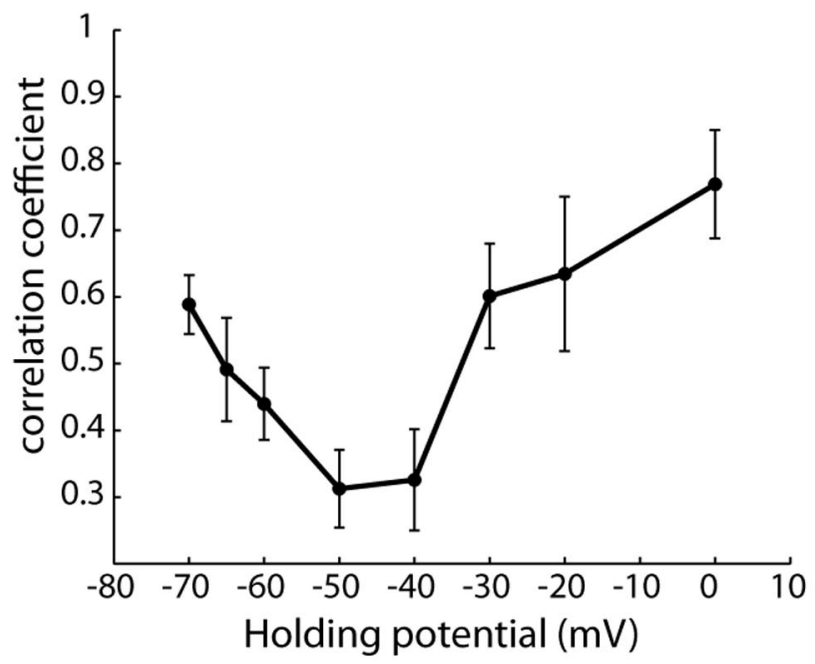

D

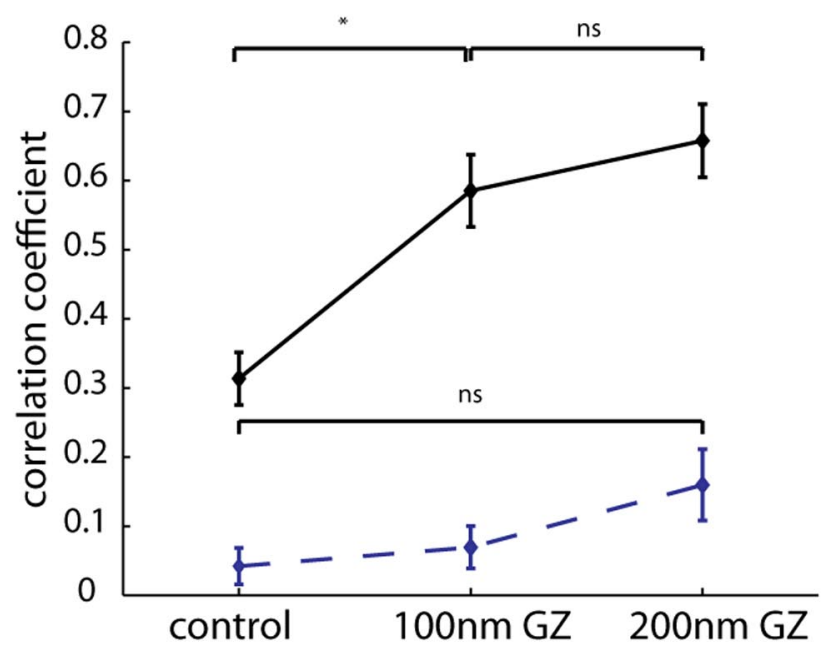

Figure 11. Pharmacologically decreasing inhibition decorrelates excitation. $A$, Schematic showing experimental configuration. Two excitatory cells were patched within $100 \mu \mathrm{m}$ of one another while a thalamically triggered UP state was simultaneously recorded in the two cells at different holding potentials ranging from $0 \mathrm{mV}$ (mostly IPSCs) to $-70 \mathrm{mV}$ (mostly EPSCs), with a mixture of IPSCs and EPSCS recorded at intermediate potentials. B, The correlation coefficient was calculated for EPSCs at these different holding potentials. Starting from $-70 \mathrm{mV}$, correlation values decreased, hitting their lowest value at intermediate potentials, and increasing again as holding potential approached $0 \mathrm{mV}$. C, Example traces from two PCs recorded $55 \mu \mathrm{m}$ from one another. These two cells were voltage-clamped at $-70 \mathrm{mV}$ so EPSCs could be recorded. Above traces are control, and below in $200 \mathrm{~nm}$ GZ. Red lines below each set of traces indicated EPSCs in both cells that occurred within $10 \mathrm{~ms}$ of one another. $\boldsymbol{D}$, Nanomolar concentrations of GZ significantly increased correlations in EPSCs ( ${ }^{*} p<0.05$, Mann-Whitney, $n=5$ pairs). Dashed line shows correlation of shuffled data, which did not differ significantly between control and either 100 or $200 \mathrm{~nm} \mathrm{GZ}$.

and synaptically, and that such coupling can promote synchrony under certain conditions (Galarreta and Hestrin, 2001; Hu et al., 2011). In these studies spiking was induced by introducing current injections in both cells simultaneously, or by activating specific subsets of neurons using neuromodulators. These manipulations do not activate all the conductances relevant during evoked or spontaneous activity in sensory cortex. The interaction of these conductances, combined with the electrical and chemical coupling of interneurons, is important when testing whether this coupling affects synchrony; when neurons are in a high conductance state, as we and others have found is the case during UP states (Gonzalez-Burgos and Barrionuevo, 2001; Bruno and Sakmann, 2006; Watson et al., 2008) (but see Waters and Helmchen, 2006), so it is possible that gap junction and inhibitory synaptic coupling have a small functional effect. The role of gap junctions could be to act as low pass filters, synchronizing subthreshold membrane potentials, and promoting synchrony over broader time scales (Galarreta and Hestrin, 1999, 2001). Alternatively, gap junctions could play a metabolic or developmental role, by enabling cells that belong to the same differentiation program to share second messengers or signaling molecules (Yuste et al., 1992).

A limitation of our work is that it was performed in an acute slice preparation, where connections are severed, and there is no neuromodulation. Although the in vitro slice preparation is widely used to examine synaptic dynamics or plasticity and cellular studies, the question remains whether the slice preparation is appropriate for the examination of neuronal population dynamics. However, the network activity we studied here, matches well that previously reported in large scale in vivo imaging studies of sensory cortices (Kenet et al., 
2003; Petersen et al., 2003). More specifically, UP states have been widely reported in vivo where they are thought to occur in an oscillatory manner during slow-wave sleep, quiet wakefulness, and under certain types of anesthesia (Sanchez-Vives and McCormick, 2000; Steriade et al., 2001; Monier et al., 2003; Luczak et al., 2007). To more closely mimic these oscillations, and to examine what effect they may have on interneuron synchrony, we used a modified ACSF with increased potassium, and found that even during UP states occurring under these conditions, interneurons were not synchronous. In addition, high-frequency thalamic stimulation in these conditions evoked UP states closely resembling the spontaneously occurring ones, which has also been found to be the case in vivo (Luczak et al., 2007). Regardless of the functional role of UP states, they are a well established form of network activity that is preserved in slices, where they can be studied in a reduced model (MacLean et al., 2005; Poulet and Petersen, 2008; Watson et al., 2008; Xu et al., 2013). Therefore, some of the same mechanisms underlying our main results are likely to be present in vivo, even if they do not represent the full spectrum of dynamics observed in awake behaving animals.

\section{Dense inhibitory connectivity leads to synchronous IPSCs}

We found that when we voltage-clamped cells within $100 \mu \mathrm{m}$ at the reversal potentials for inhibition or excitation, isolated EPSCs and IPSCs were highly correlated, with IPSCs more correlated than EPSCs. This result is similar to findings in ferret, where the higher degree of correlation among IPSCs in dually patched cells was attributed to synchronization of PV interneurons within the gamma band (Hasenstaub et al., 2005). However, three lines of evidence lead us to conclude the high correlation we observed in IPSCs is due to a greater degree of shared inhibitory inputs among nearby cells. First, inhibitory neurons, as discussed above, do not fire more synchronously than pyramidal cells. Second, the high probability of finding a connection between parvalbumin-positive and somatostatinpositive interneurons onto pyramidal cells indicates that shared inhibitory input is likely the main mechanism responsible for IPSC correlation we observed. This finding is in agreement with previous electrophysiological and two-photon mapping studies demonstrating both SOM and PV interneuron subtypes make locally dense and unspecific connections (Fino and Yuste, 2011; Packer and Yuste, 2011). In fact when we calculated the decay constant over which the probability of $\mathrm{PV} \rightarrow \mathrm{PC}$ connections tapered off (166.8 $\mu \mathrm{m})$ (Packer and Yuste, 2011) we found it agreed well with the decay constant over which correlations tapered off (165.5 $\mu \mathrm{m})$ (Fig. 9). Finally, the conductance of each IPSC detected during spontaneous and evoked activity was comparable to the conductance of individual synapses, indicating that the IPSCs during evoked and spontaneous activity could be comprised of just a single interneuron firing, rather than a synchronous group.

\section{Inhibition as a decorrelator of neuronal circuits}

The extent of membrane potential and/or spiking correlations among nearby neurons or those sharing receptive fields varies greatly across studies, in part due the manner in which such correlations are calculated (Cohen and Kohn, 2011). In particular, low spike rates like those observed during cortical UP states tend to decrease correlations. For this reason, we used principal cells as a metric for evaluating the significance of synchrony among inhibitory subtypes, and found that correlations among interneurons did not differ from principal cells. To evaluate whether or not the principal cells had significant correlated firing, we used reshuffling techniques, which demonstrated that these cells do not fire any more synchronously than what would be expected if their spikes were randomly distrib- uted. We conclude that the firing of both inhibitory cells and principal cells during UP states is asynchronous.

Together, our findings are in good agreement with recent studies in mouse and monkey primary visual cortices showing that, even when neurons share receptive fields, they do not have high correlations. (Ecker et al., 2010; Smith and Häusser, 2010; Hofer et al., 2011). In both these studies, the authors speculated that their observations could be due to some unknown, active mechanism of decorrelation. Theoretical work has suggested this mechanism could be in part due to inhibitory inputs balancing excitatory ones, effectively canceling correlations (Renart et al., 2010). In this model, when both inhibitory and excitatory inputs are correlated, inhibition closely follows excitation, thereby reducing the correlated fluctuations, and leading to sparse spiking. This may explain why the EPSC and IPSC correlations fell off with distance (Fig. 10C), although we saw no such relationship for neuronal activity (Fig. $3 E$ ). We propose that, by preventing uncontrolled network-wide synchrony rather than promoting it, inhibition may create a background of weakly correlated spiking, as required for efficient information processing based on either firing rates or coordinated spike timing patterns (Vogels and Abbott, 2009).

\section{References}

Abbott LF, Dayan P (1999) The effect of correlated variability on the accuracy of a population code. Neural Comput 11:91-101. CrossRef Medline

Agmon A, Connors BW (1991) Thalamocortical responses of mouse somatosensory (barrel) cortex in vitro. Neuroscience 41:365-379. CrossRef Medline

Ascoli GA, Alonso-Nanclares L, Anderson SA, Barrionuevo G, BenavidesPiccione R, Burkhalter A, Buzsaki G, Cauli B, Defelipe J, Fairen A, et al. (2008) Petilla terminology: nomenclature of features of GABAergic interneurons of the cerebral cortex. Nat Rev Neurosci 9:557-568. CrossRef Medline

Bartos M, Vida I, Frotscher M, Meyer A, Monyer H, Geiger JR, Jonas P (2002) Fast synaptic inhibition promotes synchronized gamma oscillations in hippocampal interneuron networks. Proc Natl Acad Sci U S A 99:13222-13227. CrossRef Medline

Beierlein M, Gibson JR, Connors BW (2000) A network of electrically coupled interneurons drives synchronized inhibition in neocortex. Nat Neurosci 3:904-910. CrossRef Medline

Blatow M, Rozov A, Katona I, Hormuzdi SG, Meyer AH, Whittington MA, Caputi A, Monyer H (2003) A novel network of multipolar bursting interneurons generates theta frequency oscillations in neocortex. Neuron 38:805-817. CrossRef Medline

Bruno RM (2011) Synchrony in sensation. Current opinion in neurobiology 21:701-708. CrossRef Medline

Bruno RM, Sakmann B (2006) Cortex is driven by weak but synchronously active thalamocortical synapses. Science 312:1622-1627. CrossRef Medline

Buchanan KA, Blackman AV, Moreau AW, Elgar D, Costa RP, Lalanne T, Tudor Jones AA, Oyrer J, Sjöström PJ (2012) Target-specific expression of presynaptic NMDA receptors in neocortical microcircuits. Neuron 75:451-466. CrossRef Medline

Chattopadhyaya B, Di Cristo G, Higashiyama H, Knott GW, Kuhlman SJ, Welker E, Huang ZJ (2004) Experience and activity-dependent maturation of perisomatic GABAergic innervation in primary visual cortex during a postnatal critical period. J Neurosci 24:9598-9611. CrossRef Medline

Cohen MR, Kohn A (2011) Measuring and interpreting neuronal correlations. Nat Neurosci 14:811-819. CrossRef Medline

Deans MR, Gibson JR, Sellitto C, Connors BW, Paul DL (2001) Synchronous activity of inhibitory networks in neocortex requires electrical synapses containing connexin36. Neuron 31:477-485. CrossRef Medline

Di Garbo A, Panarese A, Chillemi S (2005) Gap junctions promote synchronous activities in a network of inhibitory interneurons. Biosystems 79:9199. CrossRef Medline

Ecker AS, Berens P, Keliris GA, Bethge M, Logothetis NK, Tolias AS (2010) Decorrelated neuronal firing in cortical microcircuits. Science 327:584-587. CrossRef Medline 
Feldmeyer D, Egger V, Lubke J, Sakmann B (1999) Reliable synaptic connections between pairs of excitatory layer 4 neurones within a single "barrel” of developing rat somatosensory cortex. J Physiol 521:169-190. CrossRef Medline

Ferster D, Lindström S (1985) Synaptic excitation of neurones in area 17 of the cat by intracortical axon collaterals of corticogeniculate cells. J Physiol 367:233-252. Medline

Fino E, Yuste R (2011) Dense inhibitory connectivity in neocortex. Neuron 69:1188-1203. CrossRef Medline

Galarreta M, Hestrin S (1999) A network of fast-spiking cells in the neocortex connected by electrical synapses. Nature 402:72-75. CrossRef Medline

Galarreta M, Hestrin S (2001) Spike transmission and synchrony detection in networks of GABAergic interneurons. Science 292:2295-2299. CrossRef Medline

Galarreta M, Hestrin S (2002) Electrical and chemical synapses among parvalbumin fast-spiking GABAergic interneurons in adult mouse neocortex. Proc Natl Acad Sci U S A 99:12438-12443. CrossRef Medline

Gentet LJ, Avermann M, Matyas F, Staiger JF, Petersen CC (2010) Membrane potential dynamics of GABAergic neurons in the barrel cortex of behaving mice. Neuron 65:422-435. CrossRef Medline

Gibson JR, Beierlein M, Connors BW (1999) Two networks of electrically coupled inhibitory neurons in neocortex. Nature 402:75-79. CrossRef Medline

Gibson JR, Beierlein M, Connors BW (2005) Functional properties of electrical synapses between inhibitory interneurons of neocortical layer 4 . J Neurophysiol 93:467-480. CrossRef Medline

Gonchar Y, Wang Q, Burkhalter A (2007) Multiple distinct subtypes of GABAergic neurons in mouse visual cortex identified by triple immunostaining. Front Neuroanat 1:3. CrossRef Medline

González-Burgos G, Barrionuevo G (2001) Voltage-gated sodium channels shape subthreshold EPSPs in layer 5 pyramidal neurons from rat prefrontal cortex. J Neurophysiol 86:1671-1684. Medline

Gordon JA (2011) Oscillations and hippocampal-prefrontal synchrony. Curr Opini Neurobiol 21:486-491. CrossRef Medline

Hasenstaub A, Shu Y, Haider B, Kraushaar U, Duque A, McCormick DA (2005) IPSPs carry synchronized frequency information in active cortical networks. Neuron 47:423-435. CrossRef Medline

Hertz J (2010) Cross-correlations in high-conductance states of a model cortical network. Neural Comput 22:427-447. CrossRef Medline

Hofer SB, Ko H, Pichler B, Vogelstein J, Ros H, Zeng H, Lein E, Lesica NA, Mrsic-Flogel TD (2011) Differential connectivity and response dynamics of excitatory and inhibitory neurons in visual cortex. Nat Neurosci 14:1045-1052. CrossRef Medline

Hu H, Ma Y, Agmon A (2011) Submillisecond firing synchrony between different subtypes of cortical interneurons connected chemically but not electrically. J Neurosci 31:3351-3361. CrossRef Medline

Kaminski J, Wrobel A, Kublik E (2011) Gap junction blockade eliminates supralinear summation of fast $(>200 \mathrm{~Hz})$ oscillatory components during sensory integration in the rat barrel cortex. Brain Res Bull 85:424-428. CrossRef Medline

Kenet T, Bibitchkov D, Tsodyks M, Grinvald A, Arieli A (2003) Spontaneously emerging cortical representations of visual attributes. Nature 425: 954-956. CrossRef Medline

Kozloski J, Hamzei-Sichani F, Yuste R (2001) Stereotyped position of local synaptic targets in neocortex. Science 293:868-872. CrossRef Medline

Luczak A, Barthó P, Marguet SL, Buzsáki G, Harris KD (2007) Sequential structure of neocortical spontaneous activity in vivo. Proc Natl Acad Sci U S A 104:347-352. CrossRef Medline

MacLean JN, Watson BO, Aaron GB, Yuste R (2005) Internal dynamics determine the cortical response to thalamic stimulation. Neuron 48:811-823. CrossRef Medline

Markram H, Toledo-Rodriguez M, Wang Y, Gupta A, Silberberg G, Wu C (2004) Interneurons of the neocortical inhibitory system. Nat Rev Neurosci 5:793-807. CrossRef Medline

McClurkin JW, Optican LM, Richmond BJ, Gawne TJ (1991) Concurrent processing and complexity of temporally encoded neuronal messages in visual perception. Science 253:675-677. CrossRef Medline

McGarry LM, Packer AM, Fino E, Nikolenko V, Sippy T, Yuste R (2010) Quantitative classification of somatostatin-positive neocortical interneurons identifies three interneuron subtypes. Front Neural Circuits 4:12. CrossRef Medline

Monier C, Chavane F, Baudot P, Graham LJ, Frégnac Y (2003) Orientation and direction selectivity of synaptic inputs in visual cortical neurons: a diversity of combinations produces spike tuning. Neuron 37:663-680. CrossRef Medline

Nikolenko V, Watson BO, Araya R, Woodruff A, Peterka DS, Yuste R (2008) SLM microscopy: scanless two-photon imaging and photostimulation with spatial light modulators. Front Neural Circuits 2:5. CrossRef Medline

Okun M, Lampl I (2008) Instantaneous correlation of excitation and inhibition during ongoing and sensory-evoked activities. Nat Neurosci 11: 535-537. CrossRef Medline

Oliva AA Jr, Jiang M, Lam T, Smith KL, Swann JW (2000) Novel hippocampal interneuronal subtypes identified using transgenic mice that express green fluorescent protein in GABAergic interneurons. J Neurosci 20: 3354-3368. Medline

Packer AM, Yuste R (2011) Dense, unspecific connectivity of neocortical parvalbumin-positive interneurons: a canonical microcircuit for inhibition? J Neurosci 31:13260-13271. CrossRef Medline

Petersen CC, Grinvald A, Sakmann B (2003) Spatiotemporal dynamics of sensory responses in layer $2 / 3$ of rat barrel cortex measured in vivo by voltage-sensitive dye imaging combined with whole-cell voltage recordings and neuron reconstructions. J Neurosci 23:1298-1309. Medline

Peters AJ, E.G. (eds) (1984) Cellular components of the cerebral cortex. New York: Plenium.

Poulet JF, Petersen CC (2008) Internal brain state regulates membrane potential synchrony in barrel cortex of behaving mice. Nature 454:881-885. CrossRef Medline

Renart A, de la Rocha J, Bartho P, Hollender L, Parga N, Reyes A, Harris KD (2010) The asynchronous state in cortical circuits. Science 327:587-590. CrossRef Medline

Rudy B, Fishell G, Lee S, Hjerling-Leffler J (2011) Three groups of interneurons account for nearly $100 \%$ of neocortical GABAergic neurons. Dev Neurobiol 71:45-61. CrossRef Medline

Sanchez-Vives MV, McCormick DA (2000) Cellular and network mechanisms of rhythmic recurrent activity in neocortex. Nat Neurosci 3:1027-1034. CrossRef Medline

Sanchez-Vives MV, Mattia M, Compte A, Perez-Zabalza M, Winograd M, Descalzo VF, Reig R (2010) Inhibitory modulation of cortical up states. J Neurophysiol 104:1314-1324. CrossRef Medline

Sanes JN (2003) Neocortical mechanisms in motor learning. Current Opin Neurobiol 13:225-231. CrossRef Medline

Smith SL, Häusser M (2010) Parallel processing of visual space by neighboring neurons in mouse visual cortex. Nat Neurosci 13:1144-1149. CrossRef Medline

Steriade M, Timofeev I, Grenier F (2001) Natural waking and sleep states: a view from inside neocortical neurons. J Neurophysiol 85:1969-1985. Medline

Tamás G, Buhl EH, Lörincz A, Somogyi P (2000) Proximally targeted GABAergic synapses and gap junctions synchronize cortical interneurons. Nat Neurosci 3:366-371. CrossRef Medline

Uhlhaas PJ, Roux F, Rodriguez E, Rotarska-Jagiela A, Singer W (2010) Neural synchrony and the development of cortical networks. Trends Cogn Sci 14:72-80. CrossRef Medline

Vervaeke K, Lorincz A, Gleeson P, Farinella M, Nusser Z, Silver RA (2010) Rapid desynchronization of an electrically coupled interneuron network with sparse excitatory synaptic input. Neuron 67:435-451. CrossRef Medline

Vogels TP, Abbott LF (2009) Gating multiple signals through detailed balance of excitation and inhibition in spiking networks. Nat Neurosci 12: 483-491. CrossRef Medline

Vogelstein JT, Packer AM, Machado TA, Sippy T, Babadi B, Yuste R, Paninski L (2010) Fast nonnegative deconvolution for spike train inference from population calcium imaging. J Neurophysiol 104:3691-3704. CrossRef Medline

Wang Y, Toledo-Rodriguez M, Gupta A, Wu C, Silberberg G, Luo J, Markram H (2004) Anatomical, physiological and molecular properties of Martinotti cells in the somatosensory cortex of the juvenile rat. J Physiol 561:65-90. CrossRef Medline

Waters J, Helmchen F (2006) Background synaptic activity is sparse in neocortex. J Neurosci 26:8267-8277. CrossRef Medline

Watson BO, MacLean JN, Yuste R (2008) UP states protect ongoing cortical activity from thalamic inputs. PLoS One 3:e3971. CrossRef Medline

Woodruff A, Xu Q, Anderson SA, Yuste R (2009) Depolarizing effect of 
neocortical chandelier neurons. Front Neural Circuits 3:15. CrossRef Medline

Xu H, Jeong HY, Tremblay R, Rudy B (2013) Neocortical somatostatinexpressing GABAergic interneurons disinhibit the thalamorecipient layer 4. Neuron 77:155-167. CrossRef Medline

Xu X, Roby KD, Callaway EM (2010) Immunochemical characterization of inhibitory mouse cortical neurons: three chemically distinct classes of inhibitory cells. J Comp Neurol 518:389-404. CrossRef Medline
Yuste R, Katz LC (1991) Control of postsynaptic $\mathrm{Ca}^{2+}$ influx in developing neocortex by excitatory and inhibitory neurotransmitters. Neuron 6:333344. CrossRef Medline

Yuste R, Peinado A, Katz LC (1992) Neuronal domains in developing neocortex. Science 257:665-669. CrossRef Medline 